\title{
The neuropathology of traumatic brain injury
}

\author{
ANN C. MCKEE ${ }^{*}$ and DANIEL H. DANESHVAR \\ VA Boston HealthCare System; Center for the Study of Traumatic Encephalopathy, Alzheimer's \\ Disease Center, and Departments of Neurology and Pathology, Boston University School of \\ Medicine, Boston, MA, USA
}

\section{INTRODUCTION}

\section{Severity of injury}

Traumatic brain injury (TBI) occurs when a force transmitted to the head or body results in neuropathologic damage and dysfunction. The US Department of Veterans Affairs and the Department of Defense's Clinical Practice Guideline For Management of Concussion/mTBI (version 1.0, April 2009) defines TBI as

a traumatically induced structural injury and/or physiologic disruption of brain function as a result of an external force that is indicated by new onset or worsening of at least one of the following clinical signs, immediately following the event: any period of loss of or a decreased level of consciousness (LOC), any loss of memory for events immediately before or after the injury (post-traumatic amnesia [PTA]), any alteration in mental state at the time of the injury (confusion, disorientation, slowed thinking, etc.), neurologic deficits (weakness, loss of balance, change in vision, praxis, paresis/plegia, sensory loss, aphasia, etc.) that may or may not be transient, or intracranial lesion. (VA DoD, 2009)

The severity of TBI can be quantified using a variety of measures. The most commonly used assessment scales, the Glasgow Coma Scale (GCS) score and the duration of LOC or PTA, use clinical assessment to grade the severity of the trauma. Although prognosis is often predicted based on measured clinical severity, the extent to which each of these severity assessments correlates with outcome is less clear. All grades of TBI can be associated with long-term physical, emotional, behavioral, and cognitive consequences that permanently affect an individual's ability to perform routine activities and return to work (Langlois et al., 2006; Daneshvar et al. 2011b).

The GCS is used to grade TBI as mild, moderate, or severe (Table 4.1) (Teasdale and Jennett, 1974). The major advantage of the GCS is its simplicity and its usefulness as a standardized measurement that can be used to compare outcomes across a series of patients (Teasdale and Jennett, 1974). Of all severities of TBI, an estimated $75-85 \%$ are categorized as mild TBI (mTBI) with a GCS score of 13 to 15 (CDC, 2003). Mild TBI includes concussion as well as subconcussion and some blast injuries associated with improvised

"Correspondence to: Ann C. McKee, MD, VA Boston HealthCare System, 150 South Huntington Ave, Boston, MA 02130, USA. Tel: +1-857-364-5707, Fax: +1-857-364-6193, amckee@bu.edu. 
explosive devices. Mild TBI occurs in a wide variety of activities including sports, such as boxing, American football, rugby, soccer, cheerleading, ice hockey, and wrestling; military service; and in association with other exposures such as poorly controlled epilepsy, head banging, and physical abuse (Daneshvar et al., 2011a). There is often full neurologic recovery after mTBI; however, 15-30\% of subjects develop prolonged neurocognitive and behavioral changes (Daneshvar et al., 2011b) (Ch. 10). In the US, it is estimated that 1.6-3.8 million concussions occur annually (Langlois et al., 2006). Concussion is especially frequent in American football, where $4.5 \%$ of high school, $6.3 \%$ of collegiate, and $6.6 \%$ of professional football players are diagnosed with at least one concussion per season (Pellman et al., 2004; Daneshvar et al., 2011a). The true frequency of concussion is likely far greater because concussions are routinely under-recognized, under-reported and typically resolve spontaneously without medical care.

In moderate traumatic brain injury (GCS 9-13), the patient is initially lethargic or stuporous, and in severe TBI (GCS 3-8), the patient is comatose, unable to open the eyes or follow commands. Patients with severe TBI are at high risk for secondary brain injury including hypotension, hypoxemia, and brain swelling (Hukkelhoven et al., 2006). In the lower ranges of GCS score (3-9), primarily associated with severe TBI, there is a direct linear relation to a poor outcome, including severe neurologic disability, vegetative state, and death. Advancing age, particularly over the age of 60 years, is also associated with an increased risk of a poor outcome (Hukkelhoven et al., 2006) (Ch. 9).

The severity of TBI can also be categorized by the duration of LOC and PTA, measures that are reported to have better correlation with patient outcome than GCS (Forde et al., 2014). MTBI is defined as LOC of less than 1 hour and PTA for less than 24 hours, moderate TBI is defined by LOC between 1 and 24 hours or PTA for 1-7 days, and severe TBI by LOC for more than 24 hours or PTA for more than 1 week (Forde et al., 2014).

\section{Focal versus diffuse injury}

TBIs are classified as focal or diffuse based on the presence or absence of focal lesions. Although injuries may be considered predominantly focal or diffuse, most injuries are heterogeneous with both focal and diffuse components (Greenfield et al., 2008). Mass lesions, such as contusion, subdural hematoma, epidural hematoma, and intraparenchymal hemorrhage are considered focal injuries, whereas diffuse injury encompasses axonal injury, hypoxic-ischemic injury, and microvascular injury that affect widely distributed anatomic regions. The mortality rate for severe focal injuries is reported to be approximately $40 \%$ and, for severe diffuse injuries, approximately 25\% (Marshall et al., 1991) (Ch. 1).

\section{Primary versus secondary injury}

Traumatic brain injuries can also be characterized based on whether they result directly or indirectly from the trauma. Primary injuries following TBI are those that are the direct result of the external mechanical forces producing deformation of the brain tissue and disruption of normal brain function. The types of mechanical forces involved in brain trauma include acceleration and deceleration linear forces, rotational forces, forces generated by blast winds associated with blast injury, blunt impact, and penetration by a projectile. These forces 
directly damage the neurons, axons, dendrites, glia, and blood vessels in a focal, multifocal, or diffuse pattern and initiate a dynamic series of complex cellular, inflammatory, mitochondrial, neurochemical, and metabolic alterations (Graham et al., 2002) (Ch. 7). The magnitude of the primary injury resulting from a traumatic impact can be modified by the use of preventative measures, such as protective equipment and helmets. However, once the trauma occurs, the immediate neurologic damage produced by the primary traumatic forces is usually not alterable. The secondary neurologic damage produced by a cascade of secondary events after the primary injury has the potential to be reversible. Secondary brain injury occurs as a complication of the primary brain injury and includes ischemic and hypoxic damage, cerebral edema, raised intracranial pressure, hydrocephalus, and infection (Graham et al., 2002) (Chs 5, 14). Within hours of the initiating trauma, cellular and vasogenic fluid accumulates in the brain producing cerebral edema, elevated intracranial pressure, and cerebral ischemia. Brain dysfunction and morbidity are further increased by a reduction in cerebral blood flow or oxygen content below a threshold level or by cerebral herniation (Graham et al., 2002).

\section{ACUTE MODERATE-SEVERE TRAUMATIC BRAIN INJURY}

Specific injuries typically have consistent effects and can be distinguished based on whether they are associated primarily with focal or diffuse pathology. Injuries associated with focal pathology tend to have more varied symptoms based on the anatomy of the injury, whereas diffuse injuries produce more consistent effects.

\section{Focal injuries}

Skull Fractures-The presence of a skull fracture indicates that the impact had considerable force; however, many patients with a skull fracture will not have evidence of any serious brain injury. Furthermore, $20-30 \%$ of fatal head injuries are not associated with a fractured skull. A patient with a skull fracture following TBI is significantly more likely to have subarachnoid, subdural, or epidural hemorrhage (Tseng et al., 2011). Fractures of the base of the skull often involve the middle ear or anterior cranial fossa with leakage of spinal fluid from the ear (otorrhea) or nose (rhinorrhea) and cranial nerve damage.

Contusion and Laceration-Contusions are focal injuries that occur at locations where brain tissue comes in contact with irregular bony protuberances of the skull base, most often the inferior aspect of the frontal lobes, the frontal poles and inferior aspect of the temporal lobes. Contusions result from damage to small blood vessels and other components of the brain parenchyma that produce small hemorrhages perpendicular to the cortical surface. Contusions are generally most severe at the crests of the cerebral gyri but may extend through the cortex into the subcortical white matter as wedge-shaped areas of hemorrhage and necrosis. "Coup" contusions occur directly beneath the impact site and "contrecoup" contusions occur directly opposite the site of impact. Herniation contusions occur at the margins of brain herniation sites and are secondary to raised intracranial pressure. A laceration occurs when there is physical disruption of the brain parenchyma, and may occur in combination with contusions along the surface of the brain (Graham et al., 2002). 
Hemorrhage and hematoma-Hemorrhage and hematoma formation result from tearing of blood vessels at the moment of head impact. Bleeding into the subarachnoid space (subarachnoid hemorrhage) is the most common form of vascular injury after head trauma. Subdural hematoma is produced by inertial acceleration, causing bridging veins to be ruptured by rapid acceleration-deceleration forces. The hemorrhage may accumulate slowly and not be clinically manifest until hours or days after the initial injury when there is elevated intracranial pressure and herniation (Graham et al., 2002; Harvey and Close, 2012). Intraventricular hemorrhage and intracerebral hematomas are also common after brain trauma.

\section{Diffuse injuries}

Diffuse vascular injury-Petechial hemorrhages are frequent findings in severe TBI and are the result of rapid acceleration-deceleration forces producing shearing of capillaries. Petechial hemorrhages may coalesce into larger lesions with progressive secondary hemorrhage.

Diffuse brain swelling-Secondary brain swelling from edema and vascular congestion is a major contributor to increased intracranial pressure after TBI and often leads to distortion, shift, and herniation of the brain. Edematous swelling occurs around contusions and intracerebral hemorrhages. In children, diffuse swelling of the entire brain or hemisphere may occur after a seemingly trivial injury due to reactive rapidly progressive hyperemia. This syndrome, sometimes referred to as juvenile head trauma syndrome, is uncommon in adults (McQuillen et al., 1988). The cause of this rapid vasodilation and redistribution of blood into the brain parenchyma of children is not clear, but the process may involve a functional channelopathy, or a disturbance of ion channel subunits. Several individuals with this juvenile head trauma syndrome have been reported to have a mutation in the calcium channel subunit gene (CACNA1A) associated with familial hemiplegic migraine (Kors et al., 2001).

Diffuse axonal injury-Traumatic axonal injury (TAI) is damage to axons caused by trauma and may be focal, multifocal or diffuse (Graham et al., 2002). The human brain is particularly prone to damage from the mechanical forces associated with rotational and linear acceleration-deceleration because of the brain's large size and weight relative to the body, its gyrencephalic structure, and the high ratio of white matter to gray matter (Meaney et al., 1995). Axons in the white matter are especially susceptible to accelerationdeceleration injuries because of their viscoelastic nature, anisotropic arrangement in tracts, linear arrangement of microtubules and neurofilaments, and large surface to volume ratio of the axon membrane (axolemma) to the axoplasm (Johnson et al., 2013). Severe mechanical injury produces complete transection of the axon (primary axotomy), as well as disruption of blood vessels and glia. Less severe tissue deformations produce focal pathologic abnormalities in axons that sometimes lead to delayed secondary axotomy (Smith et al., 1999; Johnson et al., 2013). In addition, traumatic shearing forces produce increased membrane permeability, marked influx of calcium, swelling of mitochondria, disruption of microtubules, alterations in axonal transport, and accumulations of cytoskeletal components and membranous organelles - changes that become manifest as axonal swellings within 3-6 
hours. Between 6 and 12 hours after injury, axotomy and Wallerian degeneration of the distal segment become evident (Smith et al., 2013). Persistent axonal swelling and disconnection has been observed to continue for years after the initial trauma and is thought to contribute to the development of progressively greater disability in some individuals after TBI (Smith et al., 2013) (Ch. 9).

Neuropathologically in TAI, axonal retraction balls and axonal swellings are found diffusely distributed in the corpus callosum, internal capsule, cerebral white matter, fornix, midbrain, pons, medulla, and cerebellum (Greenfield et al., 2008). In severe TBI, there may also be hemorrhage in the corpus callosum and dorsolateral quadrants of the rostral brainstem. The detection of axonal injury usually depends on silver staining methods and immunocytochemical techniques using antibodies to amyloid precursor protein (APP) or neurofilament proteins. APP immunostaining is considered the most sensitive marker of axonal injury, and has been reported to identify axonal swellings within 35 minutes of injury (Hortobagyl et al., 2007). However, APP immunopositivity is not specific for traumatic injury and may be found after ischemia and other lesions. Furthermore, the amount of APP immunopositivity often substantially underestimates the amount of axonal injury after mild TBI. The heterogeneity of axonal pathology and pathologic assessment of the regional axonal injury generally require sophisticated histologic and quantitative techniques that may include electron microscopy.

Excitotoxicity and oxidative stress-In addition to the traumatic injury of axons, blood vessels, dendrites, and glial cells after trauma, there are substantial metabolic disturbances including the abrupt release of neurotransmitters, including glutamate, massive increases in intracellular calcium, glucose hypermetabolism, kinase activation, and diminished cerebral blood flow (Chs 5, 6, 7).

\section{Secondary effects of moderate-severe traumatic brain injury}

Diffuse and focal TBI are associated with secondary changes that may produce neuronal death and neurologic dysfunction. Unlike primary injuries, the extent of secondary injuries are potentially reversible and may be modified via prompt and proper treatment (Ghajar, 2000). Secondary injuries include damage due to reactive oxygen species generated from damaged tissue or insufficient oxygenation due to vascular disruption. Other secondary responses may be both beneficial and deleterious, such as neuroinflammation (Ghajar, 2000).

Vascular injury-Secondary hemorrhage is one of the most damaging secondary injuries resulting from TBI (Ghajar, 2000). Secondary hemorrhage usually occurs within hours of the TBI and results in expansion of tissue damage due to increased intracranial pressure, ischemia, hypoxia, free radical formation and induction of inflammation.

Hypoxic-ischemic injury-Hypotension, hypoperfusion, hypoxia, and ischemia are common secondary events following moderate-severe TBI (Ghajar, 2000) and are important determinants of neurologic outcome and mortality. In addition to relative reduction in cerebral blood flow after TBI, there is also an increase in cerebral glucose utilization. 
Hypometabolism-Functional neuroimaging techniques have demonstrated evidence of hypometabolism after all severities of TBI. Although the precise cause of this finding is unknown, it is thought that either TAI or impaired mitochondrial function are responsible (Lull et al., 2010) (Chs 5, 17).

Neuroinflammation-TBI initiates an endogenous and exogenous neuroinflammatory response. Immediately, there is rapid activation, proliferation, and migration of endogenous microglia to the injury site. The activated microglia change their morphology to an ameboidlike shape and secrete proinflammatory and anti-inflammatory cytokines and other neurotoxic products that generate free radicals (Morganti-Kossmann et al., 2007). Proinflammatory molecules such as interferon- $\gamma$ and lipopolysaccharide produce a classic activation, or M1, state. The M1 state is characterized by high levels of proinflammatory cytokines and oxidative metabolites that are necessary for phagocytosis, but may also lead to enhanced tissue destruction and extension of the injury. Conversely, anti-inflammatory cytokines such as interleukin 4 or 10 (IL-4, IL-10) stimulate alternative microglial phenotypes ("alternate activation" M2a state, or "acquired deactivated" M2c state) that act to suppress the destructive M1 immune response and promote repair processes after TBI (Loane et al., 2014). Neutrophils are also recruited to the area to phagocytize and clear cellular debris (Morganti-Kossmann et al., 2007).

Edema and herniation-Secondary brain swelling, including edema and congestion, combined with hemorrhage, are the major contributors to increased intracranial pressure. TBI alters the permeability of the blood-brain barrier, resulting in altered fluid homeostasis and edema that lead to distortion, shift, and herniation of the brain. In addition to the damage caused by increased intracranial pressure, the alteration of solute concentration impairs neuronal function (Marmarou, 2007).

\section{ACUTE MILDTRAUMATIC BRAIN INJURY}

\section{Concussive injury}

Concussion and subconcussion are produced by acceleration and deceleration forces on the brain, which may be linear or rotational (Meaney et al., 1995) (Ch. 9). Rapid acceleration, deceleration, or rotational forces cause the brain to elongate and deform, stretching individual neurons, glial cells, and blood vessels and altering membrane permeability. Although all cell compartments and blood vessels are affected by the injury, axons are especially vulnerable as they often extend long distances from the neuronal cell bodies. Axons may be injured even in the absence of the death of the neuron of origin (Povlishock et al., 1983; Povlishock, 1992; Wang et al., 2013). In addition to structural deformation, acceleration-deceleration forces produce a rapid release of neurotransmitters, influx of calcium, efflux of potassium, and acceleration of the cellular sodium-potassium $\left(\mathrm{Na}^{+}-\mathrm{K}^{+}\right)$ pump to maintain membrane homeostasis, requiring large increases in glucose metabolism. These changes are referred to as the "neurometabolic cascade of concussion" (Giza and Hovda, 2001) (Chs 5, 7). Post-concussive hypermetabolism in the setting of decreased cerebral blood flow produces a disparity between glucose supply and demand and a cellular energy crisis (Giza and Hovda, 2001). Pathologic studies of acute concussion and 
postconcussion syndrome (PCS) have shown multifocal TAI, microhemorrhage, astrocytosis, and perivascular clusters of activated microglia (Giza and Hovda, 2001; Wilde et al., 2008; Oni et al., 2010; McKee et al., 2014). The severity of the axonal injury is generally parallel to the severity of the TBI, with mild injury producing only microscopic multifocal axonal damage and moderate and severe TBI producing more severe, widespread axonal injury. Mild TBI produces multifocal and perivascular axonal injury in the corpus callosum, fornix, subcortical white matter, and cerebellum (McKee et al., 2014), physical changes that may contribute to the severity of symptoms after mTBI. In addition, focal perivascular accumulations of hyperphosphorylated tau (p-tau) as neurofibrillary tangles (NFTs) and neurites and TDP-43 immunopositive neurites in the white matter have also been found after concussion, suggesting that focal axonal injury may be mechanistically associated with the development of p-tau and TDP-43 pathology (McKee et al., 2014). Evidence of microhemorrhage as hemosiderin and hematoidin-laden macrophages may also be present after concussion, suggesting loss of microvascular integrity and breach of the blood-brain barrier occur after mTBI. Structural changes in the brain after concussive injury, such as TAI and microhemorrhages are best detected with diffusion tensor imaging (DTI) and susceptibility-weighted imaging (SWI), and are not detectable with conventional structural imaging studies, including computed tomography (CT) scan and magnetic resonance imaging (MRI). DTI provides information about the white matter microstructure and fiber tract integrity is emerging as a valuable tool in refining the diagnosis, prognosis, and management of mTBI (Wilde et al., 2008; Huang et al., 2009; Niogi and Mukherjee, 2010; Oni et al., 2010; Aoki et al., 2012). Other techniques that may be valuable in the evaluation of mTBI include alterations in brain activation through blood oxygen level dependent (BOLD) signals, resting state functional connectivity (Slobounov et al., 2010; Mayer et al, 2011), magnetic resonance spectroscopy (Vagnozzi et al., 2010), and SPECT imaging (Harch et al., 2012). Functional MRI (fMRI) studies have detected significant alterations in brain activation patterns after mTBI (McAllister et al., 2001; Chen et al., 2004, 2008; Pito et al., 2007; Gosselin et al., 2011) and these abnormal brain activation patterns can persist for months after injury.

\section{Blast injury}

The consequences of military blast injury were first recognized in World War I as "shell shock," although there was considerable debate at the time as to whether the symptoms of shell shock, such as headache, behavioral changes, and memory loss, had neuropathologic or psychiatric foundations. With the increased use of improvised explosive devices in modern times, blast injury is becoming an increasingly important form of TBI in civilian and military populations. Recent estimates indicate that $10-20 \%$ of the 2.5 million US military service members deployed to Iraq and Afghanistan are affected by TBI and the majority of these injuries are associated with blast exposure (Frieden and Collins, 2013). Individuals exposed to blast injury are susceptible to neurologic injury with acute and long-term neuropsychiatric and cognitive consequences. Some military veterans with history of blast exposure show neuropathologic changes of chronic traumatic encephalopathy (CTE) at autopsy (Goldstein et al., 2012), and single blast exposure in wild-type laboratory mice produces neuropathologic changes of axonal injury, neuroinflammation, microvascular injury, and abnormal tau pathology, as well as neurobehavioral abnormalities. A postmortem 
series of four military veterans with documented histories of blast exposure, three of whom were diagnosed with post-traumatic stress disorder (PTSD), showed focal neuropathologic changes of CTE, including cortical foci of perivascular tau pathology, disseminated microgliosis and astrocytosis, myelinated axonopathy, and focal neurodegeneration (McKee et al., 2013; McKee and Robinson, 2014) that was very similar to mild CTE pathology found in the brains of athletes with a history of repetitive concussive injury (Fig. 4.1). Clinical symptoms experienced by the veterans included progressive affective lability, irritability, distractability, executive dysfunction, memory disturbances, and cognitive deficits.

Blast injury is the result of the rapid transmission of an acoustic wave through the brain tissue and accompanying blast winds (Goldstein et al., 2012). Blast injuries are usually heterogeneous and may be associated with multiple other forms of TBI such as impact injury (Nakagawa et al., 2011). Kinematic analysis in experimental pure blast injury in mice has shown that blast winds produce forces similar to multiple, severe concussive impacts occurring over microseconds. Blast winds, not blast waves, are responsible for the resultant cerebral injury, whereas the blast-associated acoustic waves produce little deformation of brain tissue. Some of the traumatic effects of blast winds can be mitigated by immobilizing the head. Blast injuries may also produce diffuse or focal hemorrhage and edema as blood vessels and brain tissue rapidly contract and expand several times within a fraction of a second after a blast wind.

\section{Juvenile head trauma syndrome and second impact syndrome}

In children and young adults, minor brain trauma may occasionally produce catastrophic or fatal cerebral edema and coma. If the neurologic deterioration is associated with a single injury, it is referred to as juvenile head trauma syndrome (McQuillen et al., 1988). Second impact syndrome (SIS) occurs when an athlete experiences a mild head injury or concussion, then suffers a second head injury before the symptoms associated with the first injury have resolved, producing rapid cerebral swelling (Saunders and Harbaugh, 1984; Cantu, 1998; Logan et al., 2001; Miele et al., 2004; Mori et al., 2006; Cantu and Gean, 2010). Typically, in SIS the second head injury is only minor, and usually does not produce immediate loss of consciousness. However, within minutes of the injury, severe cerebral edema, vascular engorgement, and brain herniation develop with resultant clinical deterioration. SIS typically affects young athletes, particularly males (90\%) ranging in age from 10 to 24 years, mean age 17.9 years (Mori et al., 2006). Most athletes reported to have SIS were American football players, usually at the high school level. SIS has also been reported in association with boxing, karate, skiing, and ice hockey. SIS is thought to result from an abrupt post-traumatic loss of cerebral blood flow autoregulation and catecholamine release that create a rapid increase in intracranial blood volume and catastrophic cerebral edema (Clifton et al., 1981; Lam et al., 1997). In two-thirds of cases, a thin, acute subdural hematoma has been found on neuroimaging or at autopsy (Mori et al., 2006; Cantu and Gean, 2010), which may reflect the hyperemic state, in the absence of other major hematomas or space-occupying lesions. The relationship of SIS to juvenile head trauma syndrome, or malignant cerebral edema after mTBI, is uncertain, and both may be manifestations of the same underlying pathophysiology. 


\section{CHRONIC EFFECTS OF TRAUMATIC BRAIN INJURY}

There is increasing evidence that TBI promotes the accumulation, misfolding, and aggregation of multiple abnormal proteins associated with neurodegeneration, including tau, $\beta$-amyloid, a-synuclein, and tar DNA binding 43 proteins. A single moderate to severe TBI with LOC is associated with a two- to fourfold increased risk of dementia in later life (Health IoMCoGWa, 2009; Shively et al., 2012). The dementia is most often categorized as probable or possible Alzheimer's disease (AD) using validated clinical criteria, but very few studies included neuropathologic verification of the underlying neurodegenerative process. Studies also support a link between a remote single TBI and Parkinson's disease (PD), amyotrophic lateral sclerosis (ALS), and Creutzfeldt-Jakob disease (CJD) and repetitive mild TBI is a risk factor for a progressive tauopathy, chronic traumatic encephalopathy (CTE). Heterogeneity of the traumatic injuries most likely plays a critical role in governing the specific neurodegenerations triggered by TBI. Furthermore, survivors of TBI do not invariably develop dementia later in life (Moretti et al., 2012) and the nature and severity of the post-traumatic neurodegeneration most likely depend on multiple factors, including comorbid medical disorders, education, baseline intelligence, and genetic predisposition (Moretti et al., 2012).

\section{Cognitive deficits and mood disturbances}

Retired professional football players who sustained three or more concussions were found to report more cognitive symptoms including a threefold increase in significant memory impairment, and a fivefold increase in diagnosed mild cognitive impairment compared to retired players without a history of concussion (Guskiewicz et al., 2005). Other studies found cognitive deficits on neurologic and neuropsychological examination in retired NFL athletes (Ford et al., 2013; Hart et al., 2013; Randolph et al., 2013) that were associated with white matter pathology on DTI and FLAIR imaging and alterations in regional cerebral blood flow (Hampshire et al., 2013; Hart et al., 2013). Retired NFL players with only mild deficits in executive functioning showed hyperactivation and hypoconnectivity of the dorsolateral frontal and frontopolar cortices on fMRI (Hampshire et al., 2013). Clinical and functional impairments in cognition have also been correlated with the frequency of head impacts in high school and collegiate football players wearing helmet-mounted accelerometers (Breedlove et al., 2012; McAllister et al., 2012; Talavage et al., 2014), although not all helmet sensor studies have supported this relationship (Broglio et al., 2011; Gysland et al., 2012).

Brain trauma experienced in sport has also been linked to disturbances in mood. Retired professional football players who experienced three or more concussions reported a threefold increase in diagnosed depression (Guskiewicz et al., 2007). A follow-up survey administered 9 years later provided further evidence for a dose-response relationship between self-reported concussions and depressive symptoms later in life (Kerr et al., 2012). Neuropsychological assessment in former professional football players has confirmed the relationship between increased self-reported concussions and depression (Didehbani et al., 2013). Depression in these athletes is also associated with increased fractional anisotropy on 
DTI, as well as white matter abnormalities on structural imaging (Hart et al., 2013; Strain et al., 2013).

Progressive brain atrophy and progressive cognitive deficits have also been observed after TBI (reviewed in Moretti et al., 20012) (Ch. 44). Corkin and colleagues evaluated cognitive functioning after focal and penetrating TBI in World War II veterans. Cognition was measured at two time periods, 10 years and 30 years after the injury. Veterans with TBI showed greater decline in cognitive performance between the time periods compared to veterans without TBI (Corkin et al., 1989). Furthermore, former athletes who sustained a concussion more than 30 years previously, showed significant reductions in neuropsychological measures of episodic memory and electrophysiologic measures of frontal lobe function compared to former athletes who had never experienced a concussion (De Beaumont et al., 2009). In addition, global reductions in brain volume have been found after TBI with volume loss in the prefrontal, orbitofrontal, temporal lobes, and hippocampus (Tasker et al., 2005; Wilde et al., 2005; Trivedi et al., 2007). There is also evidence that the reduction in gray and white matter volume continues to progress long after brain injury (Bramlette and Dietrich, 2002; Sidaros et al., 2009). It is not clear whether this global reduction in brain volume is primarily due to persistent axonal degeneration, continued apoptosis, neuroinflammation, or other mechanisms (Rodriguez-Paez et al., 2005). The progressive atrophy correlates significantly with decline in intelligence (Grafman et al., 1986). Moreover, there is evidence that cognitive decline after penetrating head injury is associated with the possession of the glutamic acid decarboxylase markers, GAD1 rs11682957 and GAD1rs2241165, and that homozygosity for catechol- $O$-methyltransferase rs9332330 is linked to improved recovery of cognitive function (Raymont et al., 2008). Genetic polymorphisms in brain-derived neurotrophic factor (BDNF) may also play a key role in recovery after focal penetrating TBI (Krueger et al., 2011; Rostami et al., 2011). However, the greatest resilience factor against cognitive decline or depression after TBI is cognitive reserve (Kesler et al., 2003; Salmond et al., 2006; Fay et al., 2010; Glass et al., 2013).

\section{Alzheimer's disease}

In a meta-analysis of 15 case-control studies, males who had a single head injury associated with loss of consciousness (LOC) had a 50\% increased risk of AD dementia (Fleminger et al., 2003). In the MIRAGE study, head injury increased the risk of AD and the risk increased with increasing severity of the head injury (Krueger et al., 2011). Comparison of affected probands to their unaffected spouses yielded odds ratios (OR) for AD of 9.9\% (95\% confidence interval (CI), 6.5-15.1) for head injury with LOC and 3.1 (2.3-4.0) for head injury without LOC. A longitudinal study involving World War II navy veterans also demonstrated increased risk for AD in late life with increasing TBI severity (Corkin et al., 1989). Veterans with severe TBI (defined as LOC or PTA lasting longer than 24 hours) were four times more likely to have AD, whereas veterans with moderate TBI (defined as LOC or PTA lasting longer than 30 minutes but less than 24 hours) were twice as likely to have AD in late life compared to controls (Plassman et al., 2000). No increased risk was found for veterans who had a mild TBI (LOC or PTA less than 30 minutes). 
Some studies have suggested that TBI is associated with an earlier onset of AD. An analysis of $1283 \mathrm{TBI}$ survivors found that the time to onset of AD was significantly reduced in those who sustained a TBI compared to a control population (Nemetz et al., 1999). In the Northern Manhattan study, a history of head injury with LOC was associated with earlier onset of AD dementia, whereas head injury without LOC or amnesia was not significantly associated with earlier onset of AD dementia, and those with LOC exceeding 5 minutes were at significantly increased risk for AD (Schofield et al., 1997). Reanalysis of the Rochester Epidemiology Project data also indicated that the age of onset of AD among $31 \mathrm{AD}$ patients with head injury was 8 years earlier than the expected time of onset predicted by a lifetime method based on $689 \mathrm{AD}$ patents without head injury in the same cohort (Krueger et al., 2011).

Although most case control and longitudinal epidemiologic studies of dementia after TBI use clinical criteria (such as National Institute of Neurological and Communicative Disorders and Stroke-Alzheimer's Disease and Related Disorders Association (NINCDSADRDA)) to determine the presence of $\mathrm{AD}$, only scattered reports have confirmed the diagnosis of AD pathologically. Jellinger and colleagues examined the incidence of AD pathology in 55 consecutive autopsy cases with a history of single TBI. They found definite or probable $\mathrm{AD}$ in $21.8 \%$ that was significantly higher than the $14 \%$ expected in an agematched general population (Jellinger et al., 2001).

The pathologic link between Alzheimer's neurodegeneration and TBI may be related to the accumulation of $A \beta$ and tau proteins after trauma. $A \beta$ plaques and intra-axonal $A \beta$ deposits have been found in approximately one-third of TBI subjects dying shortly after injury (Gentleman et al., 2000; Ikonomovic et al., 2004; DeKosky et al., 2007), even in young subjects (Horsburgh et al., 2000), and A $\beta$ may continue to accumulate chronically (Johnson et al., 2010). Murine models also show transient elevation of APP and intra-axonal A $\beta$ deposits after acute TBI (Povlishock et al., 1983). Neuropathologic analysis of 18 cases of fatal TBI showed widespread axonal injury, accumulation of neurofilament protein, amyloid precursor protein (APP), A $\beta$ and a-synuclein in axonal bulbs and varicosities (Grafman et al., 1986). p-Tau protein was found to accumulate in both axons and neuronal cell bodies (Uryu et al., 2007). p-Tau immunoreactive NFTs have also been observed in young individuals within weeks to months following their last concussion (McKee et al., 2014). An autopsy study of single TBI survivors found $A \beta$ plaques and p-tau containing NFTs in onethird of subjects suggesting that AD may contribute to neurodegeneration following a single substantial traumatic injury (Rodriguez-Paez et al., 2005). In a single case, a 50-year-old woman developed progressive dementia and parkinsonism 11 years after a severe motor vehicle accident; at autopsy, her brain showed multiple neurodegenerative processes including atypical AD, Lewy body disease with exceptionally large Lewy bodies, axonopathy and TDP-43 proteinopathy.

Several investigators have studied the relationship between inheritance of an apolipoprotein $\varepsilon 4$ (APOE $\varepsilon 4$ ) allele and dementia after TBI. In the Northern Manhattan population study, a history of TBI and inheritance of an APOE $\varepsilon 4$ allele were associated with a 10 -fold increased risk of $\mathrm{AD}$, while $\mathrm{APOE} \varepsilon 4$ in the absence of TBI resulted in only a twofold increased risk (Mayeux et al., 1995). These results are in contrast to findings in the 
MIRAGE study in which head injury increased the odds of AD to a greater extent among those lacking the APOE $\varepsilon 4$ allele compared to those having one or two e4 alleles (Guo et al., 2000).

There is also evidence that APOE $\varepsilon 4$ is associated with deposition of $A \beta$ protein after head injury (Hartman et al., 2002). APOE $\varepsilon 4$ has been associated with increased severity of chronic neurologic deficits in boxers with high exposure to repetitive trauma compared to those without an APOE $\varepsilon 4$ allele (Jordan et al., 1997). In addition, inheritance of an APOE $\varepsilon 4$ allele is associated with longer periods of unconsciousness following severe blunt traumatic injury as well as poorer functional outcome (Friedman et al., 1999).

\section{Parkinson's disease}

In addition to AD, a link has been suggested between TBI and PD. In a case-control study, data from the Rochester Epidemiology Project was used to identify 196 subjects who developed PD and compared to controls matched for age and gender. The frequency of head trauma was significantly higher in PD cases compared to controls (OR 4.3). In addition, the OR for PD was substantially increased (11.0) in subjects who experienced mTBI with LOC or more severe TBI (Bower et al., 2003). Animal studies have shown that the brains of aged TBI-injured mice develop a transient increase in Aa-synuclein that is not found in shaminjured aged animals or TBI-injured young mice (Uryu et al., 2006). As parkinsonian symptoms may result from neuronal loss in the substantia nigra associated with either accumulation of Aa-synuclein in Lewy bodies, as occurs in PD, or p-tau inclusions in the form of NFTs, as occurs in CTE and AD, it is possible that multiple pathologies contribute to the increased frequency of parkinsonism following TBI (Uryu et al., 2003).

\section{Amyotrophic lateral sclerosis}

Amyotrophic lateral sclerosis (ALS) is a fatal progressive degeneration of motor neurons in the brain and spinal cord. Between $90 \%$ and $95 \%$ of ALS cases are sporadic, while gene mutations in copper/zinc superoxide dismutase 1, senataxin, dynactin, angiogenic, and TARDBP (the gene for transactive response DNA-binding protein of $43 \mathrm{kd}$ [TDP-43] on chromosome 1) account for some familial forms of the disease (Bruijn et al., 2004). ALS is pathologically characterized by motor neuron loss and corticospinal tract degeneration. In addition, remaining motor neurons in sporadic ALS often have ubiquitin and TDP-43 immunoreactive inclusion bodies that appear either as rounded or skeinlike inclusions. Although the etiology of sporadic ALS is unknown, it may well involve a complex interaction between genetic and environmental risk factors. Many environmental risk factors have been considered as possible triggers of ALS neurodegeneration, including a history of trauma to the brain and spinal cord (Chen et al., 2007; Strickland et al., 1996; Schmidt et al., 2010). Recent literature points toward a trend not only between CNS trauma and the development of ALS but also between a smaller number of years between the last injury and ALS diagnosis, and older age at the last injury and the development of ALS (Schmidt et al., 2010). In a case control study of 109 cases of ALS and 255 controls, (Chen et al., 2007) found that having experienced repeated head injuries or having been injured within the 10 years before diagnosis was associated with a more than three-fold higher risk of ALS (OR $3.1 ; 95 \%$ CI, 1.2-8.1; and OR 3.2; 95\% CI, 1.0-10.2, respectively), with a slightly elevated 
risk for the interval 11-30 years. The authors also further performed a meta-analysis of eight previous ALS studies and estimated a pooled OR of 1.7 (95\% CI, 1.3-2.2) for at least one previous head injury. Another case-control study, which was not included in the metaanalysis, reported an increased risk of ALS when the last head injury occurred at an older age and closer to the time of diagnosis (Binazzi et al., 2009).

\section{Creutzfeldt-Jakob disease}

Early case-control studies of Creutzfeldt-Jakob disease (CJD) suggested that trauma to the face and neck was a risk factor for development of the disease (Davanipour et al., 1985). However, a prospective case-control study based on European CJD surveillance failed to find a significant association (Zerr et al., 2000).

\section{Chronic traumatic encephalopathy}

CTE has been demonstrated in athletes, civilians, and military personnel involved in activities with a high risk for repetitive mild TBI, such as boxing, American football, ice hockey, military service, head-banging, poorly controlled epilepsy, and physical abuse (Guo et al., 2000; McKee et al., 2013). Although initially CTE was referred to as "punch drunk" and "dementia pugilistica" due to its strong association with boxers, over the past three decades the term more widely used in the literature is CTE, a term that encompasses trauma from a variety of sources capable of triggering the tauopathy.

Neuropathology of chronic traumatic encephalopathy-Like many neurodegenerative diseases, CTE can only be diagnosed definitively at postmortem neuropathologic examination (McKee et al., 2009, 2013; Stern et al., 2013) and no consensus criteria or biomarkers of disease currently exist that can be used to aid in the clinical diagnosis of CTE (Stern et al., 2013). Corsellis, Bruton, and Freeman-Browne first reported an extensive description of the clinical symptoms and neuropathologic findings in 15 former boxers (reviewed in McKee et al., 2009). They described a reduction in brain weight, enlargement of the lateral and third ventricles, thinning of the corpus callosum, cavum septum pellucidum with fenestrations, and scarring and neuronal loss of the cerebellar tonsils. Using histologic stains such as cresyl violet and silver methods they also described neurofibrillary degeneration of the substantia nigra and cerebral cortex, often in the absence of senile plaques. Subsequent studies using immunocytochemical techniques determined that most cases of CTE showed widespread diffuse beta amyloid (A $\beta$ ) deposits (reviewed in McKee et al., 2009). Immunocytochemistry for p-tau protein revealed that the NFTs in CTE were superficially distributed in layers II and III in the neocortical areas, unlike the preferential distribution of NFTs in layers 3 and 5 of isocortex in AD (Hof and colleagues, reviewed in McKee et al., 2009). Geddes (reviewed in McKee et al., 2009) observed that the NFTs in CTE were often strikingly arranged around small blood vessels.

\section{Gross pathologic features of chronic traumatic encephalopathy}

The most commonly observed gross pathologic features of CTE are generalized cerebral atrophy, with a predilection for the frontal, temporal, and medial temporal lobes, thalamic and hypothalamic atrophy, shrinkage of the mammillary bodies, enlargement of the lateral and third ventricles, thinning of the corpus callosum, cavum septum pellucidum, often with 
fenestrations, and pallor of the substantia nigra and locus coeruleus (Fig. 4.2) (McKee et al., $2009,2010)$. Cerebellar scarring, a feature noted often in early reports of boxers, is not a common feature of CTE in modern day athletes, although there is often microscopic evidence of CTE-related neurodegeneration in the cerebellum.

\section{Microscopic pathology of chronic traumatic encephalopathy}

Hyperphosphorylated tau-CTE is a tauopathy, and microscopically there are widespread deposits of p-tau protein as NFTs throughout the brain (McKee et al., 2009, 2013). Tau in CTE is similar biochemically to tau in AD. It is composed of both three and four microtubule binding repeat tau, with a ratio of 4:3 microtubule binding repeat tau of $\sim 1$, indistinguishable soluble and insoluble tau, and six abnormally phosphorylated tau isoforms (McKee et al., 2013; Stern et al., 2013). Two microscopic features unique to CTE, not found in another tauopathy, are that the tau pathology in CTE is (1) perivascular, (2) irregularly distributed in the depths of cortical sulci. Tau is also commonly found in the subpial astrocytes at the sulcal depths and there may be scattered tau deposition in the ependyma lining the ventricles and on the lateral surface of the brainstem. Tau positive axonal varicosities and neuropil threads are also found in the subcortical and deep white matter (Table 4.2, Figs 4.3-4.5).

\section{Stages of chronic traumatic encephalopathy: evolution of tau pathology. (Fig. 4.3)}

Stage I-In stage I CTE, tau is found in focal, perivascular clusters as NFTs, often at the sulcal depths of superior and dorsolateral superior frontal, septal or temporal cortex. The surrounding cortex is typically unremarkable although rare NFTs may be found in the deep nuclei, such as the locus coeruleus.

Stage II-In stage II CTE, multiple discrete clusters of perivascular p-tau NFTs are found in the sulcal depths, most commonly in frontal, temporal, parietal, insular and septal cortices. NFTs are also found in the superficial layers of adjacent cortex surrounding the epicenters. The nucleus basalis of Meynert and locus coeruleus show neurofibrillary degeneration. Rare NFTs may be found in entorhinal cortex, amygdala, hippocampus, thalamus, substantia nigra, and dorsal and median raphe nuclei of the midbrain.

Stage III-In stage III CTE, the medial temporal lobe structures including the hippocampus, entorhinal cortex, and amygdala show neurofibrillary degeneration, and there is widespread involvement of the frontal, temporal parietal, insula and septal cortices. Moderate densities of NFTs are found in olfactory bulbs, hypothalamus, thalamus, mammillary bodies, substantia nigra and dorsal and median raphe nuclei.

Stage IV-In stage IV CTE, there are dense NFTs in widespread regions of the brain, with prominent neuronal loss and gliosis of the neocortex and increasing tau in astrocytes. There is prominent neuronal loss in CA1 of the hippocampus and subiculum, cerebral cortices and increasing myelinated fiber and axonal loss in the white matter. Severe p-tau pathology is found in the cerebral cortex, diencephalon, basal ganglia, and brainstem and also affect white matter tracts and spinal cord. Primary visual cortex is spared (Fig. 4.4). 


\section{TDP-43 pathology}

The majority of CTE cases also demonstrate TDP-43 immunoreactive intraneuronal and intraglial inclusions and neurites (McKee et al., 2010). In early stage CTE, TDP-43 inclusions consist of neuritic threads and dot-like inclusions in subpial, perivascular, and periventricular regions; cytoplasmic neuronal inclusions are first seen in stage II disease and are characteristic of late stages where they partially colocalize with PHF-tau inclusions.

\section{Axonal pathology}

In addition to p-tau pathology, axonal pathology is also present at all stages of CTE and progresses with CTE severity. In the earliest stages of disease, distorted axonal varicosities are found in the cortex, subcortical white matter, and deep white matter tracts of the diencephalon. By stage III, severe axonal loss and pathologic profiles are found in the subcortical white matter, and are most severe in the frontal and temporal lobes. In advanced CTE, there is widespread axonal loss with frequent severely distorted axonal profiles widely distributed in the subcortical white matter (McKee et al., 2013).

\section{Inflammation}

Neuroinflammation is a consistent feature of CTE, large increases in activated microglia are found throughout the subcortical white matter, accompanied by a robust astrocytosis of the gray and white matter.

\section{Amyloid- $\beta$ peptide}

A $\beta$ deposits are found in 40-50\% of CTE cases, are significantly associated with age at death and generally are not a characteristic of early CTE, i.e., stages I and II (McKee et al., 2013). In CTE, $A \beta$ is found predominantly as low densities of diffuse plaques. This is in contrast to $\mathrm{AD}$, which is characterized by at least moderate densities of both diffuse and neuritic plaques. Amyloid angiopathy may also occur in CTE.

\section{Lewy bodies}

a-Synuclein-positive Lewy bodies are found in approximately $20 \%$ of CTE cases, and are significantly associated with the age of the subject at death (McKee et al., 2013).

Chronic traumatic encephalopathy and comorbid disease-CTE is associated with the development of other neurodegenerations, notably Lewy body disease (LBD), AD, frontotemporal lobar degeneration (FTLD), and motor neuron disease (MND) (McKee et al., 2013, 2014). In our current experience of 103 neuropathologically confirmed cases of CTE, coexistent LBD was found in $12(12 \%)$ cases, MND in 13 (13\%), AD in 15 (15\%), and FTLD in six (6\%), suggesting that either repetitive trauma or the accumulation of tau pathology in CTE provokes the deposition of other abnormal proteins involved in neurodegeneration (McKee et al., 2014).

Chronic traumatic encephalopathy with motor neuron disease-Exposure to repetitive mTBI in sports is associated with an increased risk for ALS. A study looking at cause of death in retired NFL players who played for 5 years or more found former NFL 
players had a 4.31 higher risk of developing ALS and a 3.86 higher risk of developing dementia compared to age and gender matched controls. Although the death certificates indicated diagnoses of dementia and ALS, there was no neuropathologic verification of the neurodegenerative processes, and the actual underlying diagnoses might well have included CTE and CTE with motor neuron disease (CTE-MND) (Lehman et al., 2012). In addition, ALS incidence and mortality are reported to be unusually high among professional soccer players in Italy (Chio et al., 2005). ALS mortality for Italian professional soccer players was reported to be increased 12 -fold, whereas mortality from other causes in the soccer players was lower or comparable to that of the general population (Belli and Vanacore, 2005).

Moreover, an incidence study involving 7325 Italian professional soccer players showed that the incidence of ALS was 6.5 times higher than expected (Chio et al., 2005). In our clinicopathologic case series of 116 individuals diagnosed with CTE, approximately $11 \%$ of individuals with CTE develop a progressive motor neuron disease (MND) that was clinically indistinguishable from sporadic ALS (Cantu, 1998; Bower et al., 2003; McKee et al., 2013, 2014). Most subjects (63\%) with CTE and MND presented with motor weakness, atrophy, and fasciculations and developed mild cognitive and behavioral symptoms several years after the onset of their motor disorder; the other $37 \%$ presented with cognitive deficits and executive dysfunction many years before developing symptoms of MND. Individuals who presented with motor symptoms of MND tended to have milder CTE at death (stages II-III), most likely reflecting their shortened lifespan, whereas those who presented with cognitive symptoms died with advanced CTE (stage III and IV). In all cases, there was a distinct TDP-43 and FUS proteinopathy affecting the spinal cord (Fig. 4.5).

Furthermore, CTE is increasingly considered an environmentally acquired frontotemporal lobar degeneration (FTLD) as CTE and FTLD share many clinical and pathologic features. CTE is accompanied by a range of clinical symptoms reflective of frontotemporal dysfunction, including behavioral and cognitive deficits and a dysexecutive syndrome. CTE is also associated with pathologic changes of frontotemporal lobar atrophy, superficial spongiosus, neuronal loss, and deposition of tau and TDP-43 proteins. In addition, a subset of CTE is associated with MND. In many respects, CTE is similar to another environmentally acquired FTLD, the amyotrophic lateral sclerosis-parkinsonism-dementia complex of Guam or Lytico-bodig disease; however tau, and TDP-43 pathology in Lyticobodig disease is not perivascular and does not have predilection for the sulcal depths.

Clinical aspects of chronic traumatic encephalopathy-Generally, CTE is characterized clinically by progressive decline of memory and executive functioning, mood and behavioral disturbances that eventually progress to dementia over the course of several decades (McKee et al., 2009, 2013; Baugh et al., 2012; Stern et al., 2013). The cognitive symptoms of CTE include episodic memory impairment and executive dysfunction, symptoms that are commonly observed late in the disease course (McKee et al., 2013; Stern et al., 2013). Mood and behavioral disturbances are also associated with CTE and include depression, apathy, impulsivity, anger, irritability, suicidal behavior, and aggressiveness (McKee et al., 2009, 2013; Stern et al., 2013) (Ch. 38). Individuals who present with mood or behavior disturbances generally develop these difficulties at mid-life and later develop cognitive symptoms as the disease advances. At the present time, there are no validated 
clinical criteria to aid the clinician in differentiating CTE from AD or any other neurodegeneration associated with aging. In addition, since the course of CTE is so protracted, long-standing behavioral symptoms of CTE may be misinterpreted as a component of the subject's premorbid personality.

\section{Clinical progression of chronic traumatic encephalopathy}

Early-stage CTE is often asymptomatic, and the symptoms that may be present, including headache, loss of attention and concentration, depression, and irritability, are nonspecific (Daneshvar et al., 2011b). Subjects with stage II CTE may demonstrate short-term memory difficulties, aggressive tendencies, difficulties with planning and organization, mood swings and explosivity (McKee et al., 2013; Stern et al., 2013). Stage II disease is also associated with paranoia and suicidality. Advanced stages of CTE (stage III and IV) are associated with cognitive impairment and memory loss (McKee et al., 2010; Stern et al., 2013). Other symptoms associated with stage III CTE include aggression, suicidal tendencies, and visuospatial difficulties (McKee et al., 2010, 2013). By stage IV CTE, executive dysfunction and memory loss are prominent features and dementia is the rule. Most individuals also exhibit profound loss of attention and concentration, language difficulties, explosivity, aggression, paranoia, depression, gait and visuospatial difficulties. Less common symptoms include impulsivity, dysarthria, and parkinsonism (McKee et al., 2010, 2013; Stern et al., 2013). A recent case series of athletes with neuropathologically confirmed CTE suggested that there might be two distinct presentations of CTE: one with a younger age at onset as behavioral disturbances (e.g., impulsivity, violence) or mood changes (e.g., depression, hopelessness), and the other that typically manifests later in life as cognitive impairment (e.g., episodic memory deficits, executive dysfunction) (Stern et al., 2013).

\section{Dementia in chronic traumatic encephalopathy}

Demented subjects with neuropathologically diagnosed stage IV CTE often meet clinical criteria for AD established by the National Institute on Aging-Alzheimer's Association (NIA-AA) Workgroup and the NINCDS-ADRDA (Stern et al., 2013). Current research is focused on delineating aspects of the clinical presentation that could help distinguish CTE from $\mathrm{AD}$, in addition to the history of neurotrauma.

\section{Chronic traumatic encephalopathy and post-traumatic stress disorder}

The clinical course of CTE (and TBI) also frequently overlaps with post-traumatic stress disorder (PTSD). Both conditions are characterized by neuropsychiatric symptoms indicative of frontal lobe dysfunction including alterations in working memory, planning, multitasking, complex decision making, judgment, empathy, executive function, impulsivity, emotional lability, and disinhibition, as well as changes in personality and social behavior. Some individuals diagnosed with PTSD have been shown to have neuropathologic changes of CTE at autopsy (Goldstein et al., 2012), and it is possible that CTE may underlie some of the symptoms that are associated with PTSD (Ch. 39). 


\section{Differential diagnosis of chronic traumatic encephalopathy}

In early stage CTE, the major differential diagnosis is prolonged PCS or PTSD. However, PCS develops immediately after the injury and diminishes or does not progress with time (Daneshvar et al., 2011b), whereas CTE usually becomes symptomatic years after the traumatic injuries and slowly advances. Later in life, CTE with cognitive impairment must be distinguished from AD or frontotemporal dementia (FTD) (Daneshvar et al., 2011b). In general, the cognitive impairments in CTE begin before the typical onset of sporadic AD and progress over many decades (McKee et al., 2013; Stern et al., 2013). Instances of CTE that demonstrate mid-life behavior and personality changes might be mistaken for FTD, but the course of the illness is far slower than would be expected in FTD.

\section{FUTURE AREAS FOR RESEARCH}

Other than trauma, the risk factors for CTE remain unknown. Future efforts to discover how gender, low cognitive reserve, drug or steroid use, and genetics contribute to the development of CTE are clearly warranted. Moreover, CTE remains a diagnosis that can only be made after death; accordingly, there is an urgent need to develop reliable biomarkers for detection and diagnosis during life. Reliable biomarkers for CTE would provide the potential to monitor putative therapies and rehabilitative strategies, as well as to guide comprehensive multidisciplinary care services for patients and their families.

\section{Potential risk factors for chronic traumatic encephalopathy}

Apolipoprotein E-Allelic variation in APOE in boxers and professional football players following a single TBI has been implicated in prolonged recovery time and lower cognitive performance (Jordan et al., 1997). Several studies reported a higher than expected prevalence of APOE $\varepsilon 4$ carriers and homozygotes in individuals with neuropathologically confirmed CTE, suggesting that APOE $\varepsilon 4$ may increase the risk of developing CTE (McKee et al., 2009, 2013; Stern et al., 2013), although no genes have been established as risk factors for CTE. Other candidate genes include MAPT and TARDBP (Czell et al., 2013; Lee et al., 2013).

\section{MAPT}

The discovery of highly-penetrant tau gene (MAPT) mutations in rare families with FTLD demonstrated that tau dysfunction alone was sufficient to cause neurodegeneration (Gasparini et al., 2007). Some MAPT mutations, clustered around exon 10, influence splicing, leading to accumulation of tau having four microtubule binding domain repeats (4R) over those with three (3R) (Vandrocova et al., 2010). MAPT also harbors a common genetic variation that is associated with tauopathy. MAPT is contained within a $\sim 900 \mathrm{~kb}$ ancestral genomic inversion that defines two haplotypes, H1 and $\mathrm{H} 2$ (Stefansson et al., 2005). These haplotypes are in complete linkage disequilibrium and do not recombine. Sporadic tauopathies such as progressive supranuclear palsy and corticobasal degeneration as well as PD are associated with the H1 haplotype (Baker et al., 1999; Di Maria et al., 2000; Bekris et al., 2010). It remains to be determined if the $\mathrm{H} 1$ haplotype pays a role in susceptibility for CTE. 
Gender-Gender differences may play a role in the development of CTE. To date, most neuropathologically confirmed cases have been identified in men; however, this likely reflects the demographics of mTBI. Women appear to be at greater risk for mTBI and PCSrelated symptoms (Daneshvar et al., 2011a). These differences may based on hormonal differences, neck and muscle strength, or simply more reporting of somatic symptoms (Daneshvar et al., 2011a). Any potential increased risk of mTBI in women may possibly influence the risk of developing CTE.

Cognitive reserve-Cognitive reserve is thought to play a significant role in the development and clinical course of CTE. In other neurodegenerative diseases such as AD, cognitive reserve is considered protective against clinical manifestations (Stern et al., 2013) and it is suggested that one can increase one's "reserve" and relative resistance with occupational and educational attainment (Stern et al., 2013) (Ch. 43). In diseases such as AD and CTE, cognitive reserve provides an explanation for individual differences in measured cognitive deficits despite similar degrees of neuropathology (Stern et al., 2013).

Biomarkers-The lack of biomarkers for clinical diagnosis hampers the ability to detect and monitor the clinical course, assess efficacy of therapies and rehabilitation techniques and assess prognosis. Furthermore, without a reliable method for clinical diagnosis, the exact incidence and prevalence of CTE cannot be easily determined.

\section{Candidate biomarkers for chronic traumatic encephalopathy}

Acute traumatic injury is associated with increased cerebrospinal fluid (CSF) levels of glial and neuronal markers. Elevations in the glial marker S100B have been consistently observed in CSF following minor head injury (Ingebrigtsen et al., 1995, 2000; Romner et al., 2000). A study of Olympic boxers found elevations of S100B, glial fibrillary acidic protein (GFAP) and the axonal markers, neurofilament light chain protein, and total tau in CSF (Neselius et al., 2012). Whether these bio-markers will have specificity or utility in diagnosing CTE is not known (Ch. 16).

Other potential biomarkers include APP and A $\beta$. A $\beta$ levels increase in the CSF following severe traumatic brain injury (Olssom et al., 2004). However, the elevations in A $\beta$ are transient and most CTE patients lack amyloid plaques. Future studies of diffusible soluble $\mathrm{A} \beta$ species such as diffusible oligomers, or alterations in other APP-derived fragments may be more informative (Walsh and Selkoe, 2007). Markers of neuroinflammation may also be helpful, as there is a marked activation of microglia/macrophages in CTE, however, lack of specificity may be problematic.

Abnormalities in tau are the most consistent feature found in CTE and tau remains the lead candidate for bio-markers. P-tau, the major disease protein in CTE, is not elevated in the acute or subacute stages following mTBI in humans (Neselius et al., 2012), yet it may be chronically elevated in CTE as it is in AD and other tauopathies (Craig-Schapiro et al., 2009). While the precise processes in the brain that drive tau accumulation and CTE are still under debate, there is a growing consensus that shearing of brain structures, including axons, serves as the trigger. Axons are rich in tau, where it binds and stabilizes microtubules (Weingarten et al., 1975), suggesting a direct molecular link between traumatic axonal 
shearing and tau dysfunction. Moreover, morphologic data supports focusing on tau as a modulator of disease severity and progression.

Identifying biomarkers in blood or other less intrusively collected body fluids would have advantages over CSF. Saliva-based biomarkers may be useful, as p-tau is detectable in saliva from patients with AD (Shi et al., 2011). Several groups have investigated plasma/serum markers in AD but tau species are not elevated (Song et al., 2009). Tau is elevated in serum during acute trauma, but whether it is elevated in serum in CTE has not been tested (Liliang et al., 2010).

\section{Blood}

Plasma provides another potential biomarker for CTE. Specifically, blood-brain barrier disruption subsequent to head injury may result in abnormal levels of many plasma proteins. Moreover, the perivascular deposition of PHF-tau characteristic of CTE suggests chronic blood-brain barrier disruption. Recently, Neselius and colleagues reported that tau was significantly increased in the plasma of boxers after a boxing bout compared to controls and the levels of tau decreased significantly after a period of rest (Neselius et al., 2013). Another recent study showed that concussed hockey players had total tau levels in their blood that were increased significantly compared to their preseason values, and these total tau levels decreased during rehabilitation (Shahim et al., 2014). Studies such as these suggest that biomarkers for axonal injury and tau may become useful blood biomarkers for acute concussive injury in the future.

Magnetic resonance imaging-Macroscopic neuropathologic changes observed in late stage CTE include cavum septum pellucidum and septal perforations, alterations that would be detectable with structural MRI.

\section{Functional magnetic resonance imaging}

Functional MRI has been used in the diagnosis of multiple neurodegenerative diseases including AD, FTD, and DLB, and may provide utility in the diagnosis of CTE (Baugh et al., 2012; Stern et al., 2013). Repetitive head impacts sustained by high school and college football players are strongly associated with changes on fMRI (Breedlove et al., 2012). Although these findings represent acute changes associated with subconcussive injury, it is not known whether they resolve with time or persist chronically as would be expected in CTE.

\section{Diffusion tensor imaging}

DTI is sensitive to diffuse axonal injury, an indicator of TBI, which has been implicated in the pathogenesis of CTE (Blennow et al., 2012); DTI may therefore serve as an early marker of CTE. Preliminary evidence has indicated changes in fractional anisotropy in former professional athletes subjected to repetitive brain trauma, compared to normal controls (Baugh et al., 2012). 


\section{Magnetic resonance spectroscopy}

Magnetic resonance spectroscopy (MRS) utilizes clinical MR scanners to noninvasively measure in vivo brain biochemical metabolites, including $\mathrm{N}$-acetyl-aspartate (NAA), creatine (Cr), choline (Cho), glutamate and glutamine (Glx), and myo-inositol (mI) (Lin et al., 2012). Several studies reported changes in these metabolites after single and repeated mTBI that correlated with clinical symptoms (Lin et al., 2012). To date, a single pilot study found significant increases in Cho and Glx in former professional athletes with a history of repetitive brain trauma as compared to age-matched controls (Lin et al., 2010).

\section{Susceptibility weighted imaging}

Susceptibility weighted imaging (SWI) reveals breakdown of the blood-brain barrier by detecting heme iron and small microhemorrhages (Baugh et al., 2012). As microvascular pathology is a component of CTE pathology, SWI may prove to be useful in the diagnosis of CTE (Baugh et al., 2012).

Positron emission tomography-Positron emission tomography (PET) is a noninvasive diagnostic imaging modality using isotope-labeled molecular probes that bind to biomolecules with high specificity and affinity. A number of investigative PET ligands designed to target $A \beta$ plaques in $A D$ have advanced to various stages of clinical evaluations including [18 F]-FDDNP, [11C]PIB, and [18 F] Florbetapir (Klunk et al., 2004; Clark et al., 2011; Tauber et al., 2013). However, most cases of CTE do not show substantial A $\beta$ pathology; indeed, all cases of early stage CTE (stage I-II) to date have been A $\beta$ negative (McKee et al., 2013). PET ligands that specifically target p-tau are currently in development. A pilot study of five former professional football players suggested that [18 F]-FDDNP might have utility in detection of CTE (Small et al., 2013); however, the pattern of abnormality found with [18 F]-FDDNP is quite different than the pattern of p-tau pathology found in CTE and there is no evidence that [18 F]-FDDNP specifically identifies p-tau. The recently identified ligands [18 F]-T807 and [18 F]-T808 have shown high binding affinity and good selectivity for p-tau aggregates in vitro and ex vivo in rodent brains (Chien et al., 2013). Pilot PET scans of humans with mild cognitive impairment or AD showed patterns of radio-tracer accumulation that correlated with cognitive impairment, mimicking the progression of p-tau pathology in AD (Chien et al., 2013). Given that p-tau in CTE is biochemically similar to p-tau in $\mathrm{AD}$, and CTE $\mathrm{p}$-tau and $\mathrm{AD}$ p-tau consist of three and four repeat tau, [F18]-T807 and [F18]-T808 are promising potential biomarkers of CTE (McKee et al., 2009, 2013).

\section{CONCLUSIONS}

TBI, particularly mTBI, has been largely overlooked as a major health concern until very recently. It is increasingly clear that TBI is a process and not a static injury, and that prolonged symptoms in TBI survivors represent functional and structural damage. Although much remains unknown about the pathogenetic mechanisms underlying neurodegeneration after mTBI, including CTE, promising efforts are underway to identify novel biomarkers to diagnose and monitor the course of disease in living subjects. Effective treatment of mTBI and concussion will need to address the diagnosis and acute care of concussion as well as 
the long-term progressive neurodegeneration that occasionally follow repetitive injury. Currently, the best therapy is prevention of traumatic injury and continued public education about proper detection and management.

\title{
ACKNOWLEDGMENTS
}

\begin{abstract}
We gratefully acknowledge the use of resources and facilities at the Edith Nourse Rogers Memorial Veterans Hospital (Bedford, MA). We also gratefully acknowledge the help of all members of the Center for the Study of Traumatic Encephalopathy at Boston University and the Boston VA, and the individuals and families whose participation and contributions made this work possible. This work was supported by the Department of Veterans Affairs; Veterans Affairs Biorepository (CSP 501); Translational Research Center for Traumatic Brain Injury and Stress Disorders (TRACTS) Veterans Affairs Rehabilitation Research and Development Traumatic Brain Injury Center of Excellence (B6796-C); National Institute of Neurological Diseases and Stroke 1U01NS086659-01, National Institute of Aging Boston University Alzheimer's Disease Center (P30AG13846; supplement 0572063345-5); National Institute of Aging Boston University Framingham Heart Study R01 (AG1649); Sports Legacy Institute; National Operating Committee on Standards for Athletic Equipment. This work was also supported by an unrestricted gift from the National Football League, the Andlinger Foundation and the WWE.
\end{abstract}

\section{References}

Aoki Y, Inokuchi R, Gunshin M, et al. Diffusion tensor imaging studies of mild traumatic brain injury: a meta-analysis. J Neurol Neurosurg Psychiatry. 2012; 83:870-876. [PubMed: 22797288]

Baker M, Litvan I, Houlden H, et al. Association of an extended haplotype in the tau gene with progressive supranuclear palsy. Hum Mol Genet. 1999; 8:711-715. [PubMed: 10072441]

Baugh CM, Stamm JM, Riley DO, et al. Chronic traumatic encephalopathy: neurodegeneration following repetitive concussive and subconcussive brain trauma. Brain Imaging Behav. 2012; 6:244-254. [PubMed: 22552850]

Bekris LM, Mata IF, Zabetian CP. The genetics of Parkinson disease. J Geriatr Psychiatry Neurol. 2010; 23:228-242. [PubMed: 20938043]

Belli S, Vanacore N. Proportionate mortality of Italian soccer players: is amyotrophic lateral sclerosis an occupational disease? Eur J Epidemiol. 2005; 20:237-242. [PubMed: 15921041]

Binazzi A, Belli S, Uccelli R, et al. An exploratory case-control study on spinal and bulbar forms of amyotrophic lateral sclerosis in the province of Rome. Amyotroph Lateral Scler. 2009; 10:361-369. [PubMed: 19922125]

Blennow K, Hardy J, Zetterberg H. The neuropathology and neurobiology of traumatic brain injury. Neuron. 2012; 76:886-899. [PubMed: 23217738]

Bower JH, Maraganore DM, Peterson BJ, et al. Head trauma preceding PD: a case-control study. Neurology. 2003; 60:1610-1615. [PubMed: 12771250]

Bramlett HM, Dietrich WD. Quantitative structural changes in white and gray matter 1 year following traumatic brain injury in rats. Acta Neuropathol (Berl). 2002; 103:607-614. [PubMed: 12012093]

Breedlove EL, Robinson M, Talavage TM, et al. Biomechanical correlates of symptomatic and asymptomatic neurophysiological impairment in high school football. J Biomech. 2012; 45:12651272. [PubMed: 22381736]

Broglio SP, Eckner JT, Surma T, et al. Post-concussion cognitive declines and symptomatology are not related to concussion biomechanics in high school football players. J Neurotrauma. 2011; 28:2061-2068. [PubMed: 21644811]

Bruijn LI, Miller TM, Cleveland DW. Unraveling the mechanisms involved in motor neuron degeneration in ALS. Annu Rev Neurosci. 2004; 27:723-749. [PubMed: 15217349]

Cantu RC. Second-impact syndrome. Clin Sports Med. 1998; 17:37-44. [PubMed: 9475969]

Cantu R, Gean A. Second impact syndrome in a small SDH: an uncommon catastrophic result of repetitive head injury with a characteristic imaging appearance. J Neurotrauma. 2010; 27:15571564. [PubMed: 20536318]

CDC. Report to Congress on mild traumatic brain injury in the United States: steps to prevent a serious public health problem. National Center for Injury Prevention and Control; Atlanta, GA: 2003. 
Chen JK, Johnston KM, Frey S, et al. Functional abnormalities in symptomatic concussed athletes: an fMRI study. Neuroimage. 2004; 22:68-82. [PubMed: 15109998]

Chen H, Richard M, Sandler DP, et al. Head injury and amyotrophic lateral sclerosis. Am J Epidemiol. 2007; 166:810-816. [PubMed: 17641152]

Chen JK, Johnston KM, Petrides M, et al. Recovery from mild head injury in sports: evidence from serial functional magnetic resonance imaging studies in male athletes. Clin J Sport Med. 2008; 18:241-247. [PubMed: 18469565]

Chien DT, Bahri S, Szardenings AK, et al. Early clinical PET imaging results with the novel PHF-tau radioligand [F-18]-T807. J Alzheimers Dis. 2013; 34:457-468. [PubMed: 23234879]

Chio A, Benzi G, Dossena M, et al. Severely increased risk of amyotrophic lateral sclerosis among Italian professional football players. Brain. 2005; 128:472-476. [PubMed: 15634730]

Clark CM, Schneider JA, Bedell BJ, et al. Use of florbetapir-PET for imaging beta-amyloid pathology. JAMA. 2011; 305:275-283. [PubMed: 21245183]

Clifton GL, Ziegler MG, Grossman RG. Circulating catecholamines and sympathetic activity after head injury. Neurosurgery. 1981; 8:10-14. [PubMed: 7207763]

Corkin S, Rosen TJ, Sullivan EV, et al. Penetrating head injury in young adulthood exacerbates cognitive decline in later years. J Neurosci. 1989; 9:3876-3883. [PubMed: 2585058]

Craig-Schapiro R, Fagan AM, Holtzman DM. Biomarkers of Alzheimer's disease. Neurobiol Dis. 2009; 35:128-140. [PubMed: 19010417]

Czell D, Andersen PM, Morita M, et al. Phenotypes in Swiss patients with familial ALS carrying TARDBP mutations. Neurodegener Dis. 2013; 12:150-155. [PubMed: 23327806]

Daneshvar DH, Nowinski CJ, McKee AC, et al. The epidemiology of sport-related concussion. Clin Sports Med. 2011a; 30:1-17. vii. [PubMed: 21074078]

Daneshvar DH, Riley DO, Nowinski CJ, et al. Long-term consequences: effects on normal development profile after concussion. Phys Med Rehabil Clin N Am. 2011b; 22:683-700. ix. [PubMed: 22050943]

Davanipour Z, Alter M, Sobel E, et al. Creutzfeldt-Jakob disease: possible medical risk factors. Neurology. 1985; 35:1483-1486. [PubMed: 3897896]

De Beaumont L, Theoret H, Mongeon D, et al. Brain function decline in healthy retired athletes who sustained their last sports concussion in early adulthood. Brain. 2009; 132:695-708. [PubMed: 19176544]

DeKosky ST, Abrahamson EE, Ciallella JR, et al. Association of increased cortical soluble abeta42 levels with diffuse plaques after severe brain injury in humans. Arch Neurol. 2007; 64:541-544. [PubMed: 17420316]

Di Maria E, Tabaton M, Vigo T, et al. Corticobasal degeneration shares a common genetic background with progressive supranuclear palsy. Ann Neurol. 2000; 47:374-377. [PubMed: 10716259]

Didehbani N, Munro Cullum C, Mansinghani S, et al. Depressive symptoms and concussions in aging retired NFL players. Arch Clin Neuropsychol. 2013; 28:418-424. [PubMed: 23644673]

DoD VA. Management of concussion-mild traumatic brain injury (mTBI). Department of Veterans Affairs; Washington, DC: 2009. VA DoD clinical practice guidelines.. Available from: http:// www.healthquality.va.gov/management_of_concussion_mtbi.asp

Fay TB, Yeates KO, Taylor HG, et al. Cognitive reserve as a moderator of postconcussive symptoms in children with complicated and uncomplicated mild traumatic brain injury. J Int Neuropsychol Soc. 2010; 16:94-105. [PubMed: 19835663]

Fleminger S, Oliver DL, Lovestone S, et al. Head injury as a risk factor for Alzheimer's disease: the evidence 10 years on; a partial replication. J Neurol Neurosurg Psychiatry. 2003; 74:857-862. [PubMed: 12810767]

Ford JH, Giovanello KS, Guskiewicz KM. Episodic memory in former professional football players with a history of concussion: an event-related functional neuroimaging study. J Neurotrauma. 2013; 30:1683-1701. [PubMed: 23679098]

Forde CT, Karri SK, Young AM, et al. Predictive markers in traumatic brain injury: opportunities for a serum biosignature. Br J Neurosurg. 2014; 28:8-15. [PubMed: 23855389] 
Frieden, TR.; Collins, MW. Department of Defense (DoD) TBI Worldwide Numbers 2000 to 2013 Q1. Washington, DC: 2013. Understanding the Public Health Problem among Current and Former Military Personnel..

Friedman G, Froom P, Sazbon L, et al. Apolipoprotein E-epsilon 4 genotype predicts a poor outcome in survivors of tramatic brain injury. Neurology. 1999; 52:244-248. [PubMed: 9932938]

Gasparini L, Terni B, Spillantini MG. Frontotemporal dementia with tau pathology. Neurodegener Dis. 2007; 4:236-253. [PubMed: 17596718]

Gentleman SM, Greenberg BD, Savage MJ, et al. A beta 42 is the predominant form of amyloid betaprotein in the brains of short-term survivors of head injury. Neuroreport. 2000; 8:1519-1522. [PubMed: 9172166]

Ghajar J. Traumatic brain injury. Lancet. 2000; 356:923-929. [PubMed: 11036909]

Giza CC, Hovda DA. The neurometabolic cascade of concussion. J Athl Train. 2001; 36:228-235. [PubMed: 12937489]

Glass L, Krueger F, Solomon J, et al. Mental paper folding performance following penetrating traumatic brain injury in combat veterans: a lesion mapping study. Cereb Cortex. 2013; 23:16631672. [PubMed: 22669970]

Goldstein LE, Fisher AM, Tagge CA, et al. Chronic traumatic encephalopathy in blast-exposed military veterans and a blast neurotrauma mouse model. Sci Transl Med. 2012; 4:134ra60.

Gosselin N, Bottari C, Chen JK, et al. Electrophysiology and functional MRI in post-acute mild traumatic brain injury. J Neurotrauma. 2011; 28:329-341. [PubMed: 21309680]

Grafman J, Salazar A, Weingartner H, et al. The relationship of brain-tissue loss volume and lesion location to cognitive deficit. J Neurosci. 1986; 6:301-307. [PubMed: 3950697]

Graham, DI.; Gennarelli, TA.; McIntosh, TK. Trauma.. In: Graham, DI.; Lantos, PL., editors. Greenfield's Neuropathology. Arnold; London: 2002. p. 823-898.

Greenfield, JG.; Love, S.; Louis, DN., et al. Greenfield's Neuropathology. 8th edn.. Hodder Arnold; London: 2008.

Guo Z, Cupples LA, Kurz A, et al. Head injury and the risk of AD in the MIRAGE study. Neurology. 2000; 54:1316-1323. [PubMed: 10746604]

Guskiewicz KM, Marshall SW, Bailes J, et al. Association between recurrent concussion and late-life cognitive impairment in retired professional football players. Neurosurgery. 2005; 57:719-726. discussion 726. [PubMed: 16239884]

Guskiewicz KM, Marshall SW, Bailes J, et al. Recurrent concussion and risk of depression in retired professional football players. Med Sci Sports Exerc. 2007; 39:903-909. [PubMed: 17545878]

Gysland SM, Mihalik JP, Register-Mihalik JK, et al. The relationship between subconcussive impacts and concussion history on clinical measures of neurologic function in collegiate football players. Ann Biomed Eng. 2012; 40:14-22. [PubMed: 21994067]

Hampshire A, Macdonald A, Owen AM. Hypoconnectivity and hyperfrontality in retired American football players. Sci Rep. 2013; 3:2972. [PubMed: 24135857]

Harch PG, Andrews SR, Fogarty EF, et al. A phase I study of low-pressure hyperbaric oxygen therapy for blast-induced post-concussion syndrome and post-traumatic stress disorder. J Neurotrauma. 2012; 29:168-185. [PubMed: 22026588]

Hart J Jr, Kraut MA, Womack KB, et al. Neuroimaging of cognitive dysfunction and depression in aging retired National Football League players: a cross-sectional study. JAMA Neurol. 2013; 70:326-335. [PubMed: 23303193]

Hartman RE, Laurer H, Longhi L, et al. Apolipoprotein E4 influences amyloid deposition but not cell loss after traumatic brain injury in a mouse model of Alzheimer's disease. J Neurosci. 2002; 22:10083-10087. [PubMed: 12451108]

Harvey LA, Close JC. Traumatic brain injury in older adults: characteristics, causes and consequences. Injury. 2012; 43:1821-1826. [PubMed: 22884759]

Health IoMCoGWa. Long-Term Consequences of Traumatic Brain Injury. Vol. 7. National Academies Press; Washington, DC: 2009. Gulf War and Health. 
Horsburgh K, Cole GM, Yang F, et al. beta-Amyloid (Abeta)42, abeta42, abeta40 and apoE immunostaining of plaques in fatal head injury. Neuropathol Appl Neurobiol. 2000; 26:124-132. [PubMed: 10840275]

Hortobagyi T, Wise S, Hunt N, et al. Traumatic axonal damage in the brain can be detected using betaAPP immunohistochemistry within 35 min after head injury to human adults. Neuropathol Appl Neurobiol. 2007; 33:226-237. [PubMed: 17359363]

Huang MX, Theilmann RJ, Robb A, et al. Integrated imaging approach with MEG and DTI to detect mild traumatic brain injury in military and civilian patients. J Neurotrauma. 2009; 26:1213-1226. [PubMed: 19385722]

Hukkelhoven CW, Rampen AJ, Maas AI, et al. Some prognostic models for traumatic brain injury were not valid. J Clin Epidemiol. 2006; 59:132-143. [PubMed: 16426948]

Ikonomovic MD, Uryu K, Abrahamson EE, et al. Alzheimer's pathology in human temporal cortex surgically excised after severe brain injury. Exp Neurol. 2004; 190:192-203. [PubMed: 15473992]

Ingebrigtsen T, Romner B, Kongstad P, et al. Increased serum concentrations of protein S-100 after minor head injury: a biochemical serum marker with prognostic value? J Neurol Neurosurg Psychiatry. 1995; 59:103-104. [PubMed: 7608699]

Ingebrigtsen T, Romner B, Marup-Jensen S, et al. The clinical value of serum S-100 protein measurements in minor head injury: a Scandinavian multicentre study. Brain Inj. 2000; 14:10471055. [PubMed: 11147577]

Jellinger KA, Paulus W, Wrocklage C, et al. Traumatic brain injury as a risk factor for Alzheimer disease. Comparison of two retrospective autopsy cohorts with evaluation of ApoE genotype. BMC Neurol. 2001; 1:3. [PubMed: 11504565]

Johnson VE, Stewart W, Smith DH. Traumatic brain injury and amyloid-beta pathology: a link to Alzheimer's disease? Nat Rev Neurosci. 2010; 11:361-370. [PubMed: 20216546]

Johnson VE, Stewart W, Smith DH. Axonal pathology in traumatic brain injury. Exp Neurol. 2013; 246:35-43. [PubMed: 22285252]

Jordan BD, Relkin NR, Ravdin LD, et al. Apolipoprotein E epsilon4 associated with chronic traumatic brain injury in boxing. JAMA. 1997; 278:136-140. [PubMed: 9214529]

Kerr ZY, Marshall SW, Harding HP Jr, et al. Nine-year risk of depression diagnosis increases with increasing self-reported concussions in retired professional football players. Am J Sports Med. 2012; 40:2206-2212. [PubMed: 22922518]

Kesler SR, Adams HF, Blasey CM, et al. Premorbid intellectual functioning, education, and brain size in traumatic brain injury: an investigation of the cognitive reserve hypothesis. Appl Neuropsychol. 2003; 10:153-162. [PubMed: 12890641]

Klunk WE, Engler H, Nordberg A, et al. Imaging brain amyloid in Alzheimer's disease with Pittsburgh Compound-B. Ann Neurol. 2004; 55:306-319. [PubMed: 14991808]

Kors EE, Terwindt GM, Vermeulen FL, et al. Delayed cerebral edema and fatal coma after minor head trauma: role of the CACNA1A calcium channel subunit gene and relationship with familial hemiplegic migraine. Ann Neurol. 2001; 49:753-760. [PubMed: 11409427]

Krueger F, Pardini M, Huey ED, et al. The role of the Met66 brain-derived neurotrophic factor allele in the recovery of executive functioning after combat-related traumatic brain injury. J Neurosci. 2011; 31:598-606. [PubMed: 21228168]

Lam JM, Hsiang JN, Poon WS. Monitoring of autoregulation using laser Doppler flowmetry in patients with head injury. J Neurosurg. 1997; 86:438-445. [PubMed: 9046300]

Langlois JA, Rutland-Brown W, Wald MM. The epidemiology and impact of traumatic brain injury: a brief overview. J Head Trauma Rehabil. 2006; 21:375-378. [PubMed: 16983222]

Lee SE, Tartaglia MC, Yener G, et al. Neurodegenerative disease phenotypes in carriers of MAPT p.A152T, a risk factor for frontotemporal dementia spectrum disorders and Alzheimer disease. Alzheimer Dis Assoc Disord. 2013; 27:302-309. [PubMed: 23518664]

Lehman EJ, Hein MJ, Baron SL, et al. Neurodegenerative causes of death among retired National Football League players. Neurology. 2012; 79:1970-1974. [PubMed: 22955124]

Liliang PC, Liang CL, Weng HC, et al. Tau proteins in serum predict outcome after severe traumatic brain injury. J Surg Res. 2010; 160:302-307. [PubMed: 19345376] 
Lin, AP.; Ramadan, S.; Box, H., et al. Neurochemical Changes in Athletes with Chronic Traumatic Encephalopathy. Radiological Society of North America; Chicago, IL: 2010.

Lin AP, Liao HJ, Merugumala SK, et al. Metabolic imaging of mild traumatic brain injury. Brain Imaging Behav. 2012; 6:208-223. [PubMed: 22684770]

Loane DJ, Kumar A, Stoica BA, et al. Progressive neurodegeneration after experimental brain trauma: association with chronic microglial activation. J Neuropathol Exp Neurol. 2014; 73:14-29. [PubMed: 24335533]

Logan SM, Bell GW, Leonard JC. Acute subdural hematoma in a high school football player after 2 unreported episodes of head trauma: a case report. J Athl Train. 2001; 36:433-436. [PubMed: 12937485]

Lull N, Noe E, Lull JJ, et al. Voxel-based statistical analysis of thalamic glucose metabolism in traumatic brain injury: relationship with consciousness and cognition. Brain Inj. 2010; 24:10981107. [PubMed: 20597637]

Marmarou A. A review of progress in understanding the pathophysiology and treatment of brain edema. Neurosurg Focus. 2007; 22:E1.

Marshall LF, Gautille T, Klauber MR, et al. The outcome of severe closed head injury. J Neurosurg. 1991; 75:S28-S36.

Mayer AR, Mannell MV, Ling J, et al. Functional connectivity in mild traumatic brain injury. Hum Brain Mapp. 2011; 32:1825-1835. [PubMed: 21259381]

Mayeux R, Ottman R, Maestre G, et al. Synergistic effects of traumatic head injury and apolipoprotein-epsilon 4 in patients with Alzheimer's disease. Neurology. 1995; 45:555-557. [PubMed: 7898715]

McAllister TW, Sparling MB, Flashman LA, et al. Differential working memory load effects after mild traumatic brain injury. Neuroimage. 2001; 14:1004-1012. [PubMed: 11697932]

McAllister TW, Flashman LA, Maerlender A, et al. Cognitive effects of one season of head impacts in a cohort of collegiate contact sport athletes. Neurology. 2012; 78:1777-1784. [PubMed: 22592370]

McKee AC, Robinson ME. Military-related traumatic brain injury and neurodegeneration. Alzheimers Dement. 2014; 10(3 Suppl.):S242-S253. [PubMed: 24924675]

McKee AC, Cantu RC, Nowinski CJ, et al. Chronic traumatic encephalopathy in athletes: progressive tauopathy after repetitive head injury. J Neuropathol Exp Neurol. 2009; 68:709-735. [PubMed: 19535999]

McKee AC, Gavett BE, Stern RA, et al. TDP-43 proteinopathy and motor neuron disease in chronic traumatic encephalopathy. J Neuropathol Exp Neurol. 2010; 69:918-929. [PubMed: 20720505]

McKee AC, Stein TD, Nowinski CJ, et al. The spectrum of disease in chronic traumatic encephalopathy. Brain. 2013; 136:43-64. [PubMed: 23208308]

McKee AC, Daneshvar DH, Alvarez VE, et al. The neuropathology of sport. Acta Neuropathol (Berl). 2014; 127:29-51. [PubMed: 24366527]

McQuillen JB, McQuillen EN, Morrow P. Trauma, sport, and malignant cerebral edema. Am J Forensic Med Pathol. 1988; 9:12-15. [PubMed: 3354517]

Meaney DF, Smith DH, Shreiber DI, et al. Biomechanical analysis of experimental diffuse axonal injury. J Neurotrauma. 1995; 12:689-694. [PubMed: 8683620]

Miele VJ, Carson L, Carr A, et al. Acute on chronic subdural hematoma in a female boxer: a case report. Med Sci Sports Exerc. 2004; 36:1852-1855. [PubMed: 15514497]

Moretti L, Cristofori I, Weaver SM, et al. Cognitive decline in older adults with a history of traumatic brain injury. Lancet Neurol. 2012; 11:1103-1112. [PubMed: 23153408]

Morganti-Kossmann MC, Satgunaseelan L, Bye N, et al. Modulation of immune response by head injury. Injury. 2007; 38:1392-1400. [PubMed: 18048036]

Mori T, Katayama Y, Kawamata T. Acute hemispheric swelling associated with thin subdural hematomas: pathophysiology of repetitive head injury in sports. Acta Neurochir Suppl. 2006; 96:40-43. [PubMed: 16671421] 
Nakagawa A, Manley GT, Gean AD, et al. Mechanisms of primary blast-induced traumatic brain injury: insights from shock-wave research. J Neurotrauma. 2011; 28:1101-1119. [PubMed: 21332411]

Nemetz PN, Leibson C, Naessens JM. Traumatic brain injury and time to onset of Alzheimer's disease: a population based study. Am J Epidemiol. 1999; 149:32-40. [PubMed: 9883791]

Neselius S, Brisby H, Theodorsson A, et al. CSF-biomarkers in Olympic boxing: diagnosis and effects of repetitive head trauma. PLoS One. 2012; 7:e33606. [PubMed: 22496755]

Neselius S, Zetterberg H, Blennow K, et al. Olympic boxing is associated with elevated levels of the neuronal protein tau in plasma. Brain Inj. 2013; 27:425-433. [PubMed: 23473386]

Niogi SN, Mukherjee P. Diffusion tensor imaging of mild traumatic brain injury. J Head Trauma Rehabil. 2010; 25:241-255. [PubMed: 20611043]

Olsson A, Csajbok L, Ost M, et al. Marked increase of beta-amyloid(1-42) and amyloid precursor protein in ventricular cerebrospinal fluid after severe traumatic brain injury. J Neurol. 2004; 251:870-876. [PubMed: 15258792]

Oni MB, Wilde EA, Bigler ED, et al. Diffusion tensor imaging analysis of frontal lobes in pediatric traumatic brain injury. J Child Neurol. 2010; 25:976-984. [PubMed: 20332386]

Pellman EJ, Powell JW, Viano DC, et al. Concussion in professional football: epidemiological features of game injuries and review of the literature - part 3. Neurosurgery. 2004; 54:81-94. discussion 96. [PubMed: 14683544]

Plassman BL, Havlik RJ, Steffens DC, et al. Documented head injury in early adulthood and risk of Alzheimer's disease and other dementias. Neurology. 2000; 55:1158-1166. [PubMed: 11071494]

Povlishock JT. Traumatically induced axonal injury: pathogenesis and pathobiological implications. Brain Pathol. 1992; 2:1-12. [PubMed: 1341941]

Povlishock JT, Becker DP, Cheng CL, et al. Axonal change in minor head injury. J Neuropathol Exp Neurol. 1983; 42:225-242. [PubMed: 6188807]

Ptito A, Chen JK, Johnston KM. Contributions of functional magnetic resonance imaging (fMRI) to sport concussion evaluation. NeuroRehabilitation. 2007; 22:217-227. [PubMed: 17917172]

Randolph C, Karantzoulis S, Guskiewicz K. Prevalence and characterization of mild cognitive impairment in retired national football league players. J Int Neuropsychol Soc. 2013; 19:873880. [PubMed: 23902607]

Raymont V, Greathouse A, Reding K, et al. Demographic, structural and genetic predictors of late cognitive decline after penetrating head injury. Brain. 2008; 131:543-558. [PubMed: 18094019]

Rodriguez-Paez AC, Brunschwig JP, Bramlett HM. Light and electron microscopic assessment of progressive atrophy following moderate traumatic brain injury in the rat. Acta Neuropathol (Berl). 2005; 109:603-616. [PubMed: 15877231]

Romner B, Ingebrigtsen T, Kongstad P, et al. Traumatic brain damage: serum S-100 protein measurements related to neuroradiological findings. J Neurotrauma. 2000; 17:641-647. [PubMed: 10972241]

Rostami E, Krueger F, Zoubak S, et al. BDNF polymorphism predicts general intelligence after penetrating traumatic brain injury. PLoS One. 2011; 6:e27389. [PubMed: 22087305]

Salmond CH, Menon DK, Chatfield DA, et al. Cognitive reserve as a resilience factor against depression after moderate/severe head injury. J Neurotrauma. 2006; 23:1049-1058. [PubMed: 16866618]

Saunders RL, Harbaugh RE. The second impact in catastrophic contact-sports head trauma. JAMA. 1984; 252:538-539. [PubMed: 6737652]

Schmidt S, Kwee LC, Allen KD, et al. Association of ALS with head injury, cigarette smoking and APOE genotypes. J Neurol Sci. 2010; 291:22-29. [PubMed: 20129626]

Schofield PW, Logroscino G, Andrews HF, et al. An association between head circumference and Alzheimer's disease in a population-based study of aging and dementia. Neurology. 1997; 49:3037. [PubMed: 9222166]

Shahim P, Tegner Y, Wilson DH, et al. Blood biomarkers for brain injury in concussed professional ice hockey players. JAMA Neurol. 2014; 71:684-692. [PubMed: 24627036] 
Shi M, Sui YT, Peskind ER, et al. Salivary tau species are potential biomarkers of Alzheimer's disease. J Alzheimers Dis. 2011; 27:299-305. [PubMed: 21841250]

Shively S, Scher AI, Perl DP, et al. Dementia resulting from traumatic brain injury: what is the pathology? Arch Neurol. 2012; 69:1245-1251. [PubMed: 22776913]

Sidaros A, Skimminge A, Liptrot MG, et al. Long-term global and regional brain volume changes following severe traumatic brain injury: a longitudinal study with clinical correlates. Neuroimage. 2009; 44:1-8. [PubMed: 18804539]

Slobounov SM, Zhang K, Pennell D, et al. Functional abnormalities in normally appearing athletes following mild traumatic brain injury: a functional MRI study. Exp Brain Res. 2010; 202:341354. [PubMed: 20039023]

Small GW, Kepe V, Siddarth P, et al. PET scanning of brain tau in retired national football league players: preliminary findings. Am J Geriatr Psychiatry. 2013; 21:138-144. [PubMed: 23343487]

Smith DH, Wolf JA, Lusardi TA, et al. High tolerance and delayed elastic response of cultured axons to dynamic stretch injury. J Neurosci. 1999; 19:4263-4269. [PubMed: 10341230]

Smith DH, Hicks R, Povlishock JT. Therapy development for diffuse axonal injury. J Neurotrauma. 2013; 30:307-323. [PubMed: 23252624]

Song F, Poljak A, Smythe GA, et al. Plasma biomarkers for mild cognitive impairment and Alzheimer's disease. Brain Res Rev. 2009; 61:69-80. [PubMed: 19464319]

Stefansson H, Helgason A, Thorleifsson G, et al. A common inversion under selection in Europeans. Nat Genet. 2005; 37:129-137. [PubMed: 15654335]

Stern RA, Daneshvar DH, Baugh CM, et al. Clinical presentation of chronic traumatic encephalopathy. Neurology. 2013; 81:1122-1129. [PubMed: 23966253]

Strain J, Didehbani N, Cullum CM, et al. Depressive symptoms and white matter dysfunction in retired NFL players with concussion history. Neurology. 2013; 81:25-32. [PubMed: 23709590]

Strickland D, Smith SA, Dolliff G, et al. Physical activity, trauma, and ALS: a case-control study. Acta Neurol Scand. 1996; 94:45-50. [PubMed: 8874593]

Talavage TM, Nauman EA, Breedlove EL, et al. Functionally-detected cognitive impairment in high school football players without clinically-diagnosed concussion. J Neurotrauma. 2014; 31:327338. [PubMed: 20883154]

Tasker RC, Salmond CH, Westland AG, et al. Head circumference and brain and hippocampal volume after severe traumatic brain injury in childhood. Pediatr Res. 2005; 58:302-308. [PubMed: 16006434]

Tauber C, Beaufils E, Hommet C, et al. Brain [18 F] FDDNP binding and glucose metabolism in advanced elderly healthy subjects and Alzheimer's disease patients. J Alzheimers Dis. 2013; 36:311-320. [PubMed: 23609763]

Teasdale G, Jennett B. Assessment of coma and impaired consciousness: a practical scale. Lancet. 1974:81-84. ii. [PubMed: 4136544]

Trivedi MA, Ward MA, Hess TM, et al. Longitudinal changes in global brain volume between 79 and 409 days after traumatic brain injury: relationship with duration of coma. J Neurotrauma. 2007; 24:766-771. [PubMed: 17518532]

Tseng WC, Shih HM, Su YC, et al. The association between skull bone fractures and outcomes in patients with severe traumatic brain injury. J Trauma. 2011; 71:1611-1614. discussion 1614. [PubMed: 22182871]

Uryu K, Giasson BI, Longhi L, et al. Age-dependent synuclein pathology following traumatic brain injury in mice. Exp Neurol. 2003; 184:214-224. [PubMed: 14637093]

Uryu K, Richter-Landsberg C, Welch W, et al. Convergence of heat shock protein 90 with ubiquitin in filamentous alpha-synuclein inclusions of alpha-synucleinopathies. Am J Pathol. 2006; 168:947961. [PubMed: 16507910]

Uryu K, Chen XH, Martinez D, et al. Multiple proteins implicated in neurodegenerative diseases accumulate in axons after brain trauma in humans. Exp Neurol. 2007; 208:185-192. [PubMed: 17826768]

Vagnozzi R, Signoretti S, Cristofori L, et al. Assessment of metabolic brain damage and recovery following mild traumatic brain injury: a multicentre, proton magnetic resonance spectroscopic study in concussed patients. Brain. 2010; 133:3232-3242. [PubMed: 20736189] 
Vandrovcova J, Anaya F, Kay V, et al. Disentangling the role of the tau gene locus in sporadic tauopathies. Curr Alzheimer Res. 2010; 7:726-734. [PubMed: 20704554]

Walsh DM, Selkoe DJ. A beta oligomers - a decade of discovery. J Neurochem. 2007; 101:1172_ 1184. [PubMed: 17286590]

Wang J, Fox MA, Povlishock JT. Diffuse traumatic axonal injury in the optic nerve does not elicit retinal ganglion cell loss. J Neuropathol Exp Neurol. 2013; 72:768-781. [PubMed: 23860030]

Weingarten MD, Lockwood AH, Hwo SY, et al. A protein factor essential for microtubule assembly. Proc Natl Acad Sci U S A. 1975; 72:1858-1862. [PubMed: 1057175]

Wilde EA, Hunter JV, Newsome MR, et al. Frontal and temporal morphometric findings on MRI in children after moderate to severe traumatic brain injury. J Neurotrauma. 2005; 22:333-344. [PubMed: 15785229]

Wilde EA, McCauley SR, Hunter JV, et al. Diffusion tensor imaging of acute mild traumatic brain injury in adolescents. Neurology. 2008; 70:948-955. [PubMed: 18347317]

Zerr I, Brandel JP, Masullo C, et al. European surveillance on Creutzfeldt-Jakob disease: a casecontrol study for medical risk factors. J Clin Epidemiol. 2000; 53:747-754. [PubMed: 10941953] 


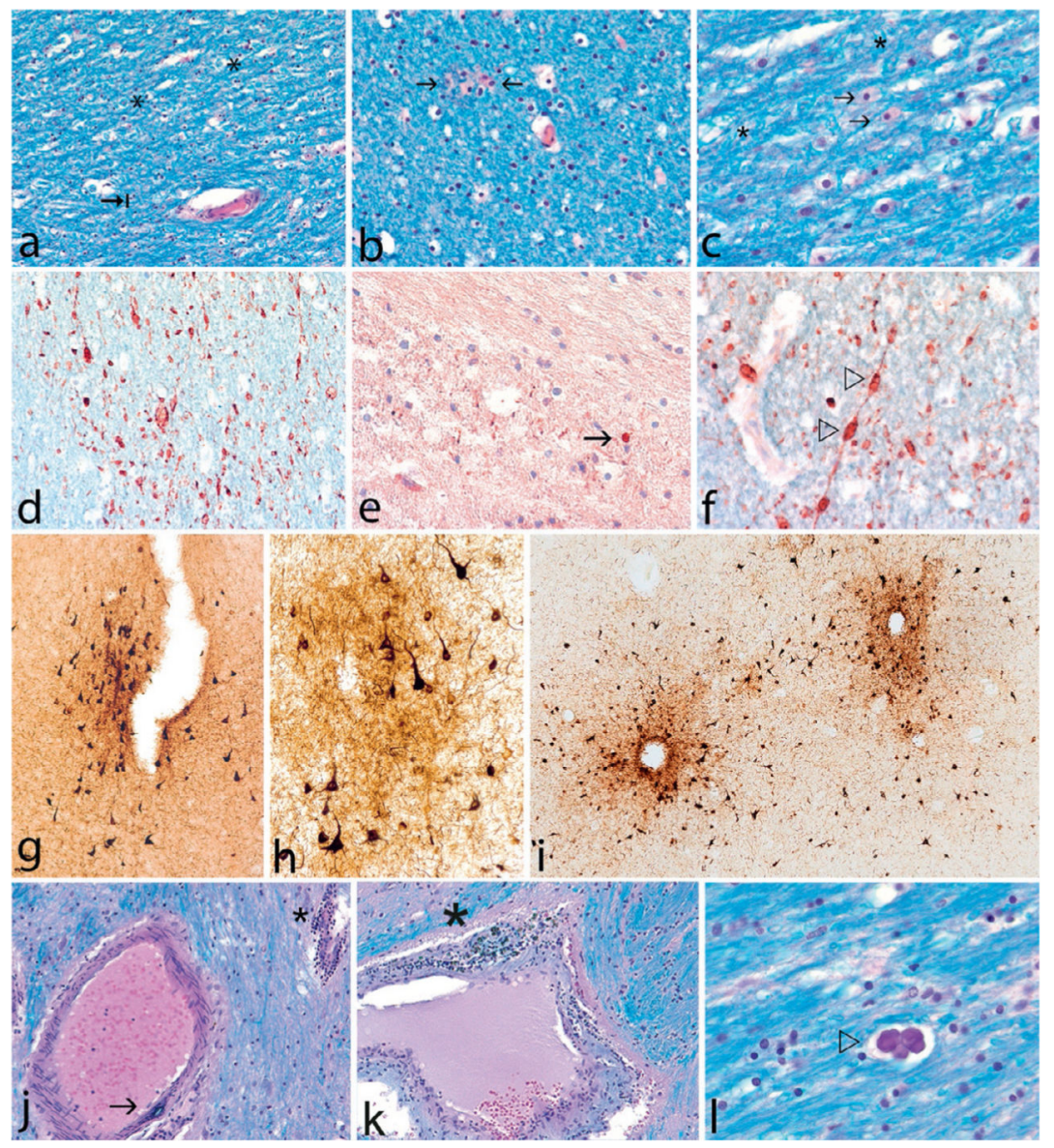

Fig. 4.1.

Neuropathologic changes associated with blast injury. (A-C) Subcortical frontal white matter shows loss of myelinated fibers, abnormal myelin clumps (asterisks), astrocytosis (arrows), luxol fast blue-hematoxylin and eosin stain, original magnification $(\mathbf{A}) \times 200,(\mathbf{B})$ $\times 400,($ C $) \times 600$. (D) Axons in the frontal white matter are severely depleted with many irregular axonal swellings, SMI-34 immunostaining, original magnification $\times 400$. (E) APP immunostaining shows axon retraction bulbs and other axonal irregularities, APP immunostain, original magnification $\times 400$. (F) Axonal swellings, SMI-34 immunostain, original magnification $\times 600$. (G) A single focus of perivascular neurofibrillary tangles (NFTs) and neurites at the depth of sulcus in inferior parietal cortex consistent with stage I/IV chronic traumatic encephalopathy (CTE), AT8 immunostaining for hyperphosphorylated tau, original magnification $\times 100$. (H) Perivascular NFTs at the sulcal depths of frontal cortex, AT8 immunostaining, original magnification $\times 200$. (I) Multiple areas of perivascular NFTs, AT8 immunostaining, original magnification $\times 100$. (J) Small blood vessels in the thalamus show prominent perivascular lymphocytic cuffing (asterisk) while a neighboring medium size artery shows focal degenerative calcification of the blood vessel wall (arrow), luxol fast blue-hematoxylin and eosin stain, original magnification $\times 200$. (K) A medium size vessel in the thalamus shows lymphocytic infiltration of the vascular wall and hemosiderin-laden macrophages (asterisk), luxol fast blue-hematoxylin and eosin stain, original magnification $\times 400$. (L) Focus of dystrophic calcification in the white matter, 
luxol fast blue-hematoxylin and eosin stain, original magnification $\times 400$. (Adapted from McKee and Robinson, 2014.) 


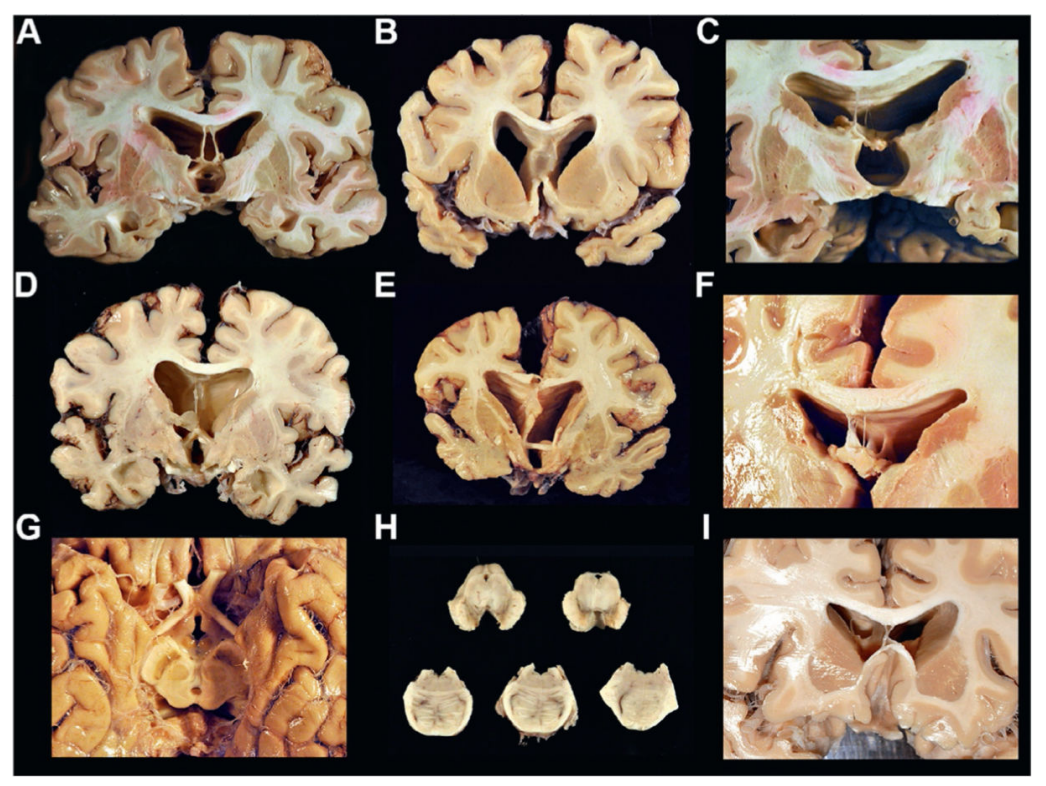

Fig. 4.2.

Gross neuropathologic features of chronic traumatic encephalopathy (CTE). In advanced CTE, there is often diffuse cerebral atrophy, marked atrophy of the medial temporal lobe structures (hippocampus, amygdala and entorhinal cortex), enlargement of the lateral and third ventricles (with disproportionate enlargement of the third ventricle), and atrophy of the diencephalon and mammillary bodies. Other features include cavum septum pellucidum ((A) and $(\mathbf{B}))$, fenestrated or torn posterior septum pellucidum $(\mathbf{C}-\mathbf{F}, \mathbf{I})$, pallor of the substantia nigra $(\mathbf{G}, \mathbf{H})$ and pallor of the locus coeruleus $(\mathbf{H})$. 
Stage I
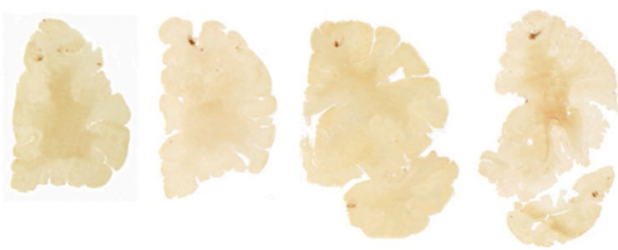

Stage II
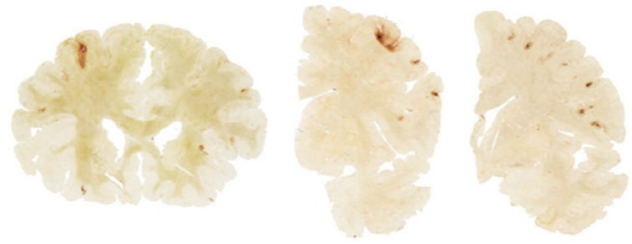

Stage III
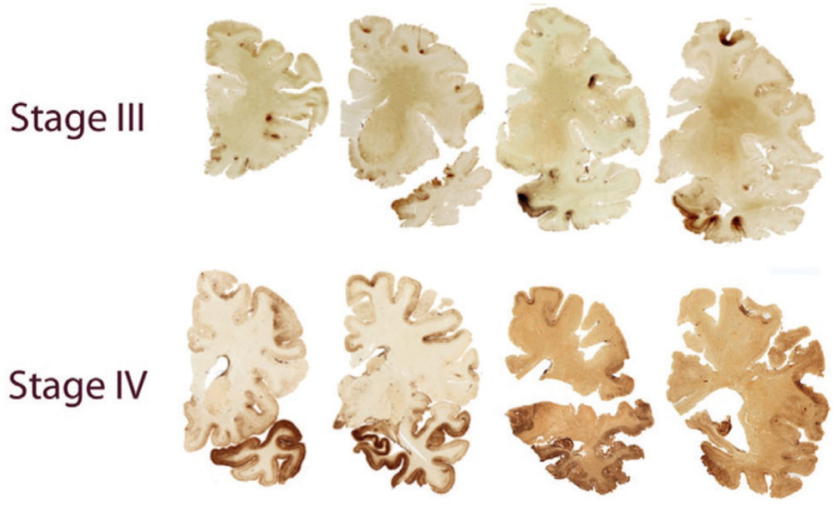

Fig. 4.3.

Staging of hyperphosphorylated tau pathology in chronic traumatic encephalopathy (CTE). In stage I CTE, hyperphosphorylated p-tau pathology is restricted to discrete foci in the cerebral cortex, most commonly in the superior, dorsolateral or lateral frontal cortices, and typically around small vessels at the depths of sulci. In stage II CTE, there are multiple epicenters at the depths of the cerebral sulci and spread of neurofibrillary pathology to the superficial layers of adjacent cortex. The medial temporal lobe is spared neurofibrillary ptau pathology in stage II CTE although it becomes progressively more involved as disease severity increases. In stage III, p-tau pathology is widespread; the frontal, insular, temporal, and parietal cortices, amygdala, hippocampus and entorhinal cortex show widespread neurofibrillary pathology. In stage IV CTE, there is widespread severe p-tau pathology affecting most regions of the cerebral cortex and the medial temporal lobe, sparing calcarine cortex in all but the most severe cases. All images, CP-13 immunostained $50 \mu$ tissue sections. Adapted from McKee et al., 2013.) 

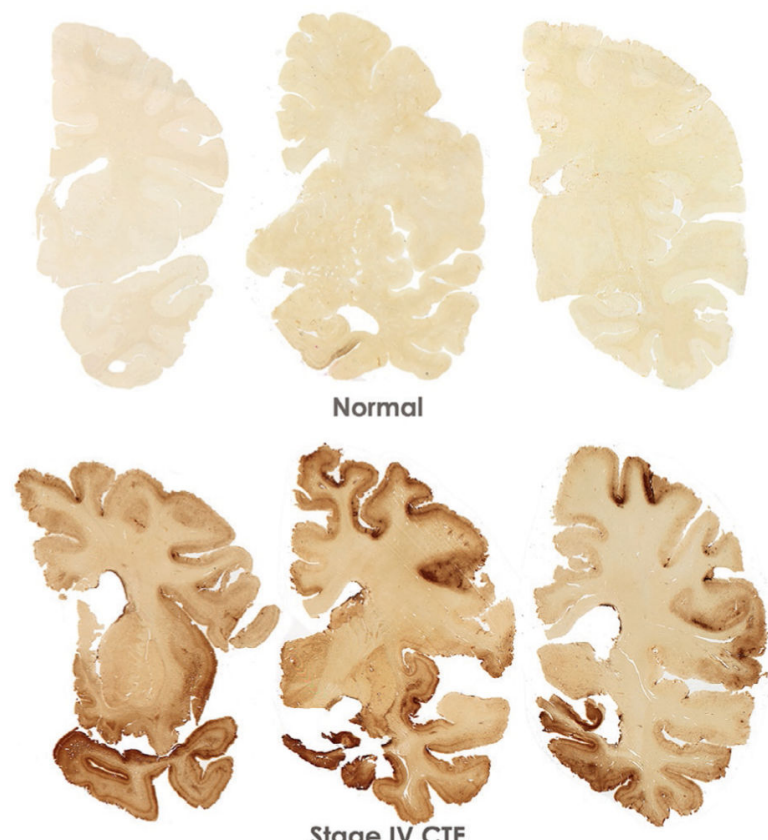

Stage IV CTE

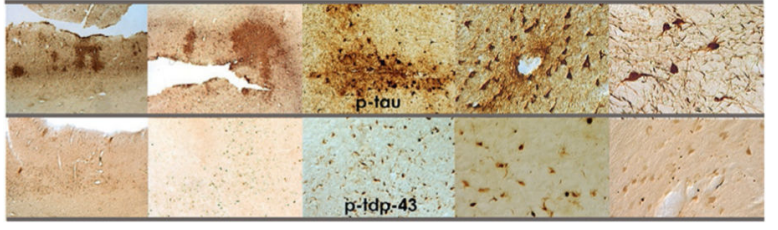

Fig. 4.4.

Microscopic features of stage IV chronic traumatic encephalopathy (CTE). (Top row) Whole mount coronal sections of the brain from cognitively intact 65 year old subject; $\mathrm{CP}-13$ immunostained $50 \mu$ tissue sections. (Second row) Whole mount $50 \mu$ sections of brain from 66-year-old with stage IV CTE. There is widespread p-tau immunoreactive neurofibrillary pathology. (Third row) Microscopic sections from 66-year-old with stage IV CTE show irregular focal patches of dense p-tau pathology, centered around small blood vessels and most severe at the depths of the sulci; CP-13 immunostaining, $10 \mu \mathrm{m}$ sections. (Fourth row) P-TDP-43 immunoreactive neuronal and glial inclusions and neurites are densely deposited in the lower layers of the frontal and temporal cortices of the 66-year-old with stage IV CTE; pTDP-43 immunostaining, $10 \mu \mathrm{m}$ sections. There is also a slight tendency for the abnormal pTDP-43 deposits to be concentrated around small blood vessels. 

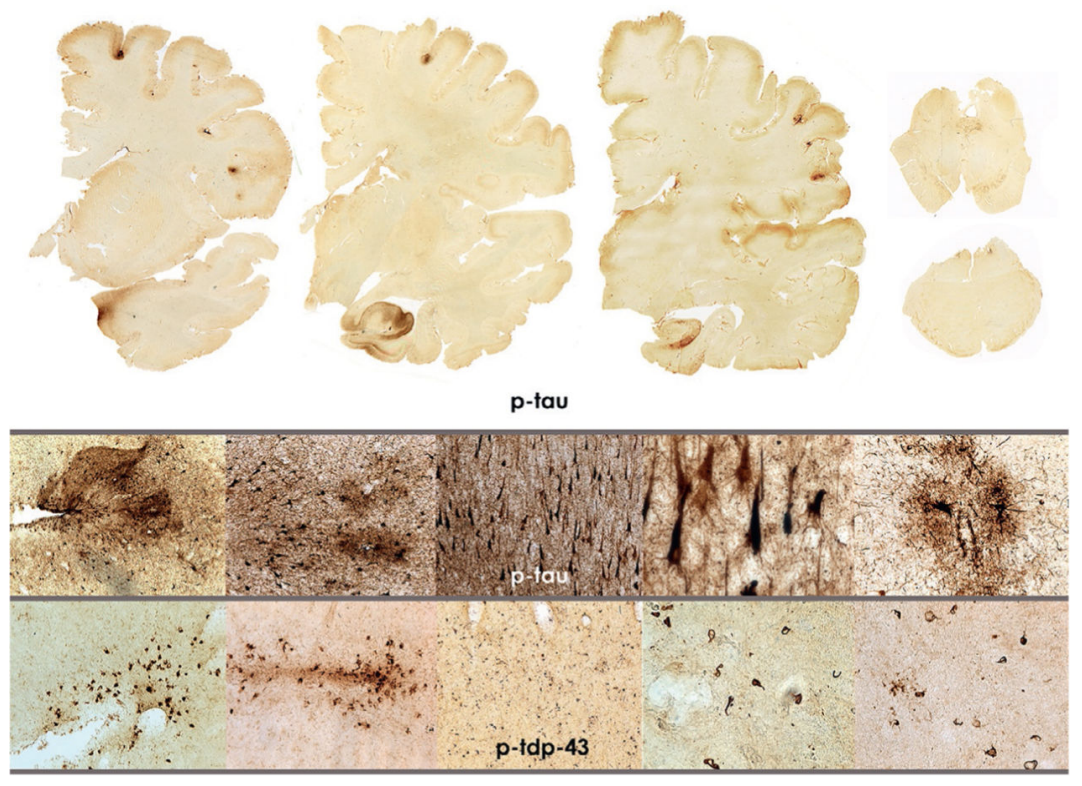

Fig. 4.5.

Chronic traumatic encephalopathy (CTE) stage II-III and motor neuron disease. (Top row) Whole mount $50 \mu$ sections of brain from 43-year-old with stage II-III CTE with motor neuron disease. P-tau immunoreactive neurofibrillary pathology is most dense in multiple foci at the depths of the sulci in the frontal lobes. There is also p-tau neurofibrillary pathology in the hippocampus and entorhinal cortex. (Second row) Microscopic sections show irregular focal patches of dense p-tau pathology in the frontal cortex, centered around small blood vessels and most severe at the depths of the sulci, as well as dense NFTs in CA1 of the hippocampus; CP-13 immunostaining, $50 \mu \mathrm{m}$ free-floating sections. (Third row) PTDP-43 immunoreactive neuronal and glial inclusions and neurites are densely deposited around blood vessels and in the subpial regions at the depths of the sulci of the frontal and temporal cortices; dense pTDP43 neuronal and glial inclusions and neurites are found in the spinal cord as well as the cerebral cortex; pTDP-43 immunostaining, $10 \mu \mathrm{m}$ sections. 
Table 4.1

Standard Glasgow Coma Scale score

\begin{tabular}{lll}
\hline Eye opening & Best verbal response & Best motor response \\
\hline $4:$ spontaneous & $5:$ oriented & $6:$ obeys commands \\
$3:$ to speech & $4:$ confused & $5:$ localizes \\
$2:$ to pain & $3:$ inappropriate words & $4:$ withdraws \\
$1:$ none & \\
& $2:$ incomprehensible sounds & $2:$ extension \\
& \\
& $1:$ none & $1:$ none \\
\hline & TOTAL GCS SCORE: $3-15$ \\
\hline
\end{tabular}

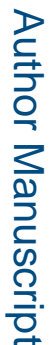

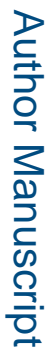


Table 4.2

Characteristics of the hyperphosphorylated tau pathology in CTE

\author{
1. Perivascular foci of $\mathrm{p}$-tau immunoreactive NFTs \\ 2. Irregular cortical distribution of p-tau immunoreactive NFTs with a predilection for the depth of cerebral sulci \\ 3. Clusters of subpial and periventricular ATs in the cerebral cortex, diencephalon, and brainstem \\ 4. NFTs in the cerebral cortex located preferentially in the superficial layers
}

Adapted from McKee et al., 2013.

NFT, neurofibrillary tangle; AT, astrocytic tangles; CTE, chronic traumatic encephalopathy. 